\title{
Sustainable Construction of
} Heterocyclic 1,2,3-Triazoles by Strict Click [3+2] Cycloaddition Reactions Between Azides and Alkynes on Copper/Carbon in Water

\author{
Noura Aflak ${ }^{1}$, Hicham Ben El Ayouchia ${ }^{1 *}$, Lahoucine Bahsis ${ }^{1}$, El Mountassir El Mouchtari ${ }^{1}$, \\ Miguel Julve ${ }^{2}$, Salah Rafqah ${ }^{1}$, Hafid Anane ${ }^{1}$ and Salah-Eddine Stiriba ${ }^{1,2 *}$ \\ ${ }^{1}$ Laboratoire de Chimie Analytique et Moléculaire, LCAM, Faculté Polydisciplinaire de Safi, Université Cadi Ayyad, Safi, \\ Morocco, ${ }^{2}$ Instituto de Ciencia Molecular/ICMol, Universidad de Valencia, Valencia, Spain
}

OPEN ACCESS

Edited by:

Oliver Reiser,

University of Regensburg, Germany

Reviewed by:

Anandarup Goswami,

Vignan's Foundation for Science,

Technology and Research, India

Markus Kaiser,

University of Duisburg-Essen,

Germany

*Correspondence:

Hicham Ben El Ayouchia belayou@gmail.com

Salah-Eddine Stiriba stiriba@uv.es

Specialty section:

This article was submitted to Green and Sustainable Chemistry,

a section of the journal

Frontiers in Chemistry

Received: 02 October 2018

Accepted: 29 January 2019

Published: 19 February 2019

Citation:

Aflak N, Ben El Ayouchia H, Bahsis L, El Mouchtari EM, Julve M, Rafqah S,

Anane H and Stiriba S-E (2019)

Sustainable Construction of Heterocyclic 1,2,3-Triazoles by Strict Click [3+2] Cycloaddition Reactions

Between Azides and Alkynes on

Copper/Carbon in Water.

Front. Chem. 7:81.

doi: 10.3389/fchem.2019.00081
1,4-Disubstituted-1,2,3-triazoles, considered as an important and useful class of heterocycles with potential applications in material science and biology, have been prepared in an efficient and selective manner by copper on carbon-catalyzed [3+2] cycloaddition reactions of azides and alkynes (CuAAC) in water under strict click chemistry conditions. Copper $(I)$ catalysts heterogenized onto commercially activated carbon materials (Cu-CC) and on another carbon material produced from vegetable biomass using Argan nut shells (Cu-CANS) were found to be versatile catalytic sources for sustainable CuAAC. These copper on carbon supports were prepared and fully characterized by using two types of activated carbons that exhibit different porosity and specific surface. The delineation of the nature of the catalytic copper species and the role of the carbon support in the CuAAC were addressed. These heterogeneous copper on carbon catalysts were recovered and reused until ten catalytic runs without any noticeable loss of activity.

Keywords: copper, activated carbon, 1, 2, 3-triazole, click chemistry, heterogeneous catalyst,
recovery/recycling, water

\section{INTRODUCTION}

1,2,3-Triazoles are important and useful non-classical bioisostere linkage heterocycles. They have several applications as agrochemical agents, dyes, corrosion inhibitors, photostabilizers, and photographic materials. Several 1,2,3-triazole derivatives show interesting biological activities under the so-called peptidomimetic substances (Chung et al., 2002; Mark, 2006; Run et al., 2007; Ostapenko et al., 2008; Zheng et al., 2008). The most popular method for the construction of 1,2,3triazole moiety is the Huisgen reaction of [3+2] dipolar cycloaddition of azides with alkynes (Yan et al., 2005; Zhang et al., 2006). Copper(I)-catalyzed azide-alkyne cycloaddition (CuAAC) (Tornøe et al., 2002; Pachón et al., 2005; Bock et al., 2006) has emerged as one of the most reliable reactions under the click chemistry regime (Kolb and Sharpless, 2003; Yadav et al., 2007) that enables the practical and efficient preparation of 1,4-disubstituted-1,2,3-triazoles, from a wide range of substrates with excellent selectivity, which cannot be achieved by traditional Huisgen non-catalyzed thermal approaches (Huisgen, 1963). 


\section{1. $\operatorname{mix}$ in $\mathrm{H}_{2} \mathrm{O}$ \\ $\mathrm{Cu}\left(\mathrm{NO}_{3}\right)_{2}+$ Carbon (Darko KB) $\stackrel{\text { 2. Ultrasound }}{\longrightarrow} \mathrm{Cu} / \mathrm{C}$ \\ 3. Distill off $\mathrm{H}_{2} \mathrm{O}$ \\ 4. Azetropic \\ drying with toluene}

SCHEME 1 | Lipshutz's protocol for the preparation of "copper-in-charcoal."

\section{1. $\mathrm{mix}$ in acetonitrile \\ 2. Stirring at r.t. \\ $\mathrm{CuI}+$ Carbon(CANS) \\ 3. Filtration \\ 4. Drying in air}

SCHEME 2 | Preparation scheme of copper on carbon support made from Argan nut shells Cu-CANS.
The synthesis of 1,2,3-triazoles under CuAAC proceeds in the presence of copper salts as homogeneous catalysts, which make the separation and recovery of such copper catalysts very difficult (Gaetke and Chow, 2003; Hong et al., 2010; Yang et al., 2014). In addition, it is most likely that under homogeneous catalytic fashion, the final 1,2,3-triazoles can be contaminated by copper particles. So far, many challenges remain and much work still needs to be done in terms of the copper catalyst recovery and the obtention of copper-free 1,2,3-triazolic compounds. Therefore, an efficient and simple way to synthesize 1,2,3-triazoles is still necessary by working under strict click chemistry conditions and taking into account the sustainable chemistry criteria. In order to overcome these problems, recent works have focused on heterogeneous catalytic systems, which have several advantages such as good dispersion of the catalytically active sites, easier and safer handling, easy separation of the products from the reaction mixture and reusability of the catalyst. Thus, a good number of heterogeneous catalytic systems has been developed. For instance, immobilizing copper salts on silica and their use as heterogeneous catalysts in CuAAC (Miao and Wang, 2008; Coelho et al., 2010; Diz et al., 2015; Jumde et al., 2015), as magnetic nanoparticles (Xiong and Cai, 2013; Moghaddam and Ayati, 2015; Pourjavadi et al., 2015, 2016; Tajbakhsh et al., 2015; Bahrami and Arabi, 2016; Jahanshahi and Akhlaghinia, 2016), polymers (Bonami et al., 2009; Wallyn et al., 2011; Xiong et al., 2016), zeolites (Chassaing et al., 2007, 2008), hydroxyapatite (Masuyama et al., 2011), and carbon support such as charcoal (Lipshutz and Taft, 2006) have been subject of previous works.

The Lipshutz group has in fact described the preparation of copper-in-charcoal by impregnating of copper(II) nitrate in activated carbon (Darco-KB) using water as solvent under ultrasound radiation followed by distillation of water by azeotropic drying with toluene. The prepared copper-in-charcoal namely $\mathrm{Cu} / \mathrm{C}$ was employed to assist the click of 1,2,3-triazoles using high temperature microwave conditions (Scheme 1).

Although the Lipshutz's copper-in-charcoal was efficient in assisting $\mathrm{CuAAC}$, the studied triazole click reactions made use of a high catalyst loading of $10 \mathrm{~mol} \%$, an additional base $\left(\mathrm{Et}_{3} \mathrm{~N}\right)$ and were peformed at high temperature $\left(60^{\circ} \mathrm{C}\right)$ in a hazardous solvent such as dioxane. The catalyst could be reused for only just three catalytic cycles without any loss of its activity (Lipshutz and Taft, 2006; Buckley et al., 2015).

In recent years, green synthesis and nature-friendly as well as sustainable resources and processes involving supported catalysts from agricultural wastes biomass have been found to be of increasing interest in the synthesis of heterocycles. In the context of our efforts to develop green, highly eco-efficient, and practical chemical methods utilizing bio-heterogeneous catalysts in [3+2] cycloaddition reactions of azides with alkynes (Bahsis et al., 2018), we herein report an easy sustainable protocol for the synthesis of 1,2,3-triazoles. For that, we use a new supported copper(I) on activated carbon materials easily made from agricultural wastes biomass such as Argan Nut Shells, namely $\mathrm{Cu} / \mathrm{CANS}$ (Scheme 2). The choice of starting from $\mathrm{CuX}$ $(\mathrm{X}=\mathrm{I}, \mathrm{Br}, \mathrm{Cl})$ precursor and its impregnation into the pores of the carbon material in acetonitrile arises from the fact that such precursor of $\mathrm{Cu}(\mathrm{I})$, considered as the catalytically active specie in $\mathrm{CuAAC}$, is nicely soluble in acetonitrile and the solutions are air stable and may be stored at room temperature.

Such a copper-supported on carbon $\mathrm{Cu}$ /CANS was found to be highly eco-efficient to assist $\mathrm{CuAAC}$ in water at room temperature as well as a recyclable heterogeneous catalyst. The commercially available activated carbon material $(\mathrm{Cu} / \mathrm{C})$ was also used for comparative purposes.

\section{EXPERIMENTAL SECTION}

\section{General Methods}

All chemicals were used as purchased without further purification. The reactions were performed under ambient conditions. NMR analyses were carried out on a spectrometer Bruker AC-400 MHz (400 MHz for proton, $100 \mathrm{MHz}$ for carbon) by using deuterated chloroform as solvent. The chemical shifts ( $\delta)$ are expressed in $\mathrm{ppm}$. The high resolution mass spectra (HRMS) were recorded in the $\mathrm{EI}(70 \mathrm{eV})$ or FAB mode at the mass spectrometry service of the University of Valencia. Melting points were determined using a Stuart melting point apparatus SMP3, employing the capillary tubes. FT-IR spectra (4000-450 $\mathrm{cm}^{-1}$ range) were recorded with a Nicolet 5700 FT-IR spectrometer on samples prepared as $\mathrm{KBr}$ pellets. The polycrystalline sample of each support was lightly ground in an agate mortar, pestle and filled into $0.5 \mathrm{~mm}$ borosilicate capillary prior to being mounted and aligned on an Empyrean PANalytical powder diffractometer using $\mathrm{Cu}-\mathrm{K} \alpha$ radiation $(\lambda$ $=1.54056 \AA)$. Three repeated measurements were collected at room temperature in the $10^{\circ}<2 \Theta<60^{\circ}$ range with a step size of $0.01^{\circ}$. Scanning Electronic Microscopy (SEM) images were obtained with a HITACHI-S4100 equipment operated at $20 \mathrm{kV}$. The specific surface areas were determined from the dinitrogen adsorption/desorption isotherms (at $77 \mathrm{~K}$ ) on a Quantachrome Autosorb-1 nautomatic analyzer using the BET (Brunauer-Emmett Teller) method. The pore size distribution was calculated from the $\mathrm{N}_{2}$ adsorption isotherms with the classic theory model of Barrett, Joyner and Halenda (BJH). 
The Scanning Electron Microscopy was carried out by using a VEGA3 TESCAN microscope and a high resolution JEOL Field Emission Gun-Scanning Electron Microscope (FEG-SEM).

\section{Procedure for the Synthesis of the Benzyl Azide Derivative}

Benzyl bromide derivative (10.0 g, $58.5 \mathrm{mmol}$ ) and $\mathrm{NaN}_{3}(11.4 \mathrm{~g}$, $175 \mathrm{mmol}$ ) were dissolved in $200 \mathrm{~mL}$ of dimethylformamide. The reaction mixture protected from light is stirred for $20 \mathrm{~h}$ at room temperature. After filtration, water was added to the filtrate and the product was extracted with dichloromethane three times. The organic phases were combined, dried over anhydrous $\mathrm{MgSO}_{4}$, then filtered-off and the solvents evaporated under reduced pressure, yielding a liquid product.

\section{Synthesis of 1-(Azidomethyl)Benzene}

Colorless liquid. Yield: $90 \% . R_{f}=0.8$ in hexane/ethyl acetate (4:1 v/v). ${ }^{1} \mathrm{H}$ NMR (400 MHz, $\left.\mathrm{CDCl}_{3}, \delta \mathrm{ppm}\right): 4.39$ (s, 2H, $\left.\mathrm{CH}_{2}\right)$; 7.41-7.5 (m, 5H, $\mathrm{CH}_{\mathrm{ar}}$ ). FT-IR (film on $\mathrm{NaCl}, \mathrm{cm}^{-1}$ ): $2,096 \mathrm{~cm}^{-1}\left(\mathrm{~N}_{3}\right)$.

\section{Synthesis of \\ (Azidomethyl)-4-Methoxybenzene}

Brown liquid. Yield: $87 \% . R_{f}=0.64$ in hexane/ethyl acetate (4:1 v/v). ${ }^{1} \mathrm{H}$ NMR (400 MHz, $\mathrm{CDCl}_{3}, \delta \mathrm{ppm}$ ): 3.68 (s, 3H, OMe); 4.13 (s, 2H, $\left.\mathrm{CH}_{2}\right)$; 6.78-6.81 (d, $\left.J=12.00 \mathrm{~Hz}, 2 \mathrm{H}, \mathrm{CH}_{\mathrm{ar}}\right) ; 7.11-$ $7.14\left(\mathrm{~d}, J=12.00 \mathrm{~Hz}, 2 \mathrm{H}, \mathrm{CH}_{\mathrm{ar}}\right) .{ }^{13} \mathrm{C} \mathrm{NMR}\left(100 \mathrm{MHz}, \mathrm{CDCl}_{3}, \delta\right.$ ppm): $54.3\left(\mathrm{CH}_{2}\right) ; 55.2\left(\mathrm{CH}_{3}\right) ; 114.2\left(2 \mathrm{CH}_{\mathrm{ar}}\right) ; 127.4\left(\mathrm{C}_{\mathrm{ar}}\right) ; 129.7$ $\left(2 \mathrm{CH}_{\mathrm{ar}}\right) ; 159.64\left(\mathrm{C}_{\mathrm{ar}}\right)$.

\section{Procedure for the Synthesis of 1-Azidobenzene}

Aniline $(13 \mathrm{mmol})$ was suspended in $80 \mathrm{~mL}$ of hydrochloric acid (17\%) at room temperature, then ethanol was added until a clear solution was obtained. The solution was cooled to $0^{\circ} \mathrm{C}$ and $\mathrm{NaNO}_{2}(19.5 \mathrm{mmol})$ was added in small portions. After stirring at $0^{\circ} \mathrm{C}$ for $15-30 \mathrm{~min}, \mathrm{NaN}_{3}(19.5 \mathrm{mmol})$ was slowly added (caution!! when handling $\mathrm{NaN}_{3}$ ) and the mixture was stirred for additional $2 \mathrm{~h}$ at room temperature. The reaction mixture was extracted with diethyl ether $(3 \times 80 \mathrm{~mL})$ and the combined organic fractions were washed with saturated $\mathrm{NaHCO}_{3}$ solution $(3 \times 50 \mathrm{~mL})$ and with brine $(50 \mathrm{~mL})$. After drying over $\mathrm{MgSO}_{4}$ the ether was removed under reduced pressure and the desired azidobenzene was obtained without further purification as a brown liquid. Yield: $90 \% . R_{f}=0.86$ in hexane. ${ }^{1} \mathrm{H}$ NMR (400 MHz, $\mathrm{CDCl}_{3}, \delta$ ppm): 7.08-7.44 (m, 5H, $\mathrm{CH}_{\mathrm{ar}}$ ). ${ }^{13} \mathrm{C}$ NMR (100 MHz, $\left.\mathrm{CDCl}_{3}, \delta \mathrm{ppm}\right): 119.46\left(\mathrm{CH}_{\mathrm{ar}}\right) ; 125.31$ $\left(2 \mathrm{CH}_{\mathrm{ar}}\right) ; 130.19\left(2 \mathrm{CH}_{\mathrm{ar}}\right) ; 140.6\left(\mathrm{C}_{\mathrm{ar}}\right)$. FT-IR (film on $\mathrm{NaCl}$, $\left.\mathrm{cm}^{-1}\right): 2,119 \mathrm{~cm}^{-1}\left(\mathrm{~N}_{3}\right)$.

\section{Procedure for the Preparation of Activated Carbon-Argan Nut Shells (CANS)}

The argan oil shells were collected, washed with distilled water, dried at room temperature and crushed by a plunger ball mill until a fine powder was obtained. The unmodified Argan nut shells material is abbreviated as ANS. The prepared raw material was treated by phosphoric acid with a mass proportion of $\mathrm{H}_{3} \mathrm{PO}_{4} /$ ANS 1:1. The mixture was stirred using the plaster mill with a speed of $400 \mathrm{rpm} / \mathrm{mn}$ for $10 \mathrm{~min}$. Subsequently, the mixture was kept at $120^{\circ} \mathrm{C}$ during $4 \mathrm{~h}$. The carbonization of the obtained dried material was carried out in a muffle furnace at $500^{\circ} \mathrm{C}$ for $1 \mathrm{~h}$ at air atmosphere, whereas the activation with phosphate group would produce a well-developed porosity of the as-prepared activated carbon. After cooling, the recovered solid was crushed, washed several times with $0.15 \mathrm{M} \mathrm{HCl}$ solution, then with distilled water until the $\mathrm{pH}$ of the solution becomes neutral $(\mathrm{pH} \sim 7$ ). The resulting material was dried completely and then crushed again by the plunger ball mill at a speed of $400 \mathrm{rpm}$ for $15 \mathrm{~min}$. Finally the obtained phosphate-containing carbon material was kept in a hermetic glass bottle.

\section{Synthetic Procedure of Copper on Carbon Catalyst}

The commercially available carbon (CC) or carbon prepared from Argan nut shells (CANS) (1 g) was added to a solution of copper(I) iodide $(250 \mathrm{mg})$ in acetonitrile $(50 \mathrm{~mL})$. The suspension was stirred overnight at room temperature and the resulting solid compounds were filtered-off, washed with acetonitrile (2 $\times 15 \mathrm{~mL})$, diethyl ether $(2 \times 15 \mathrm{~mL})$ and dried overnight. The catalyst was characterized by X-ray diffraction (XRD), scanning electronic microscopy (SEM), and infrared spectroscopy (FT-IR). Atomic Absorption Spectroscopy (AAS) (Aurora AI800) was used to determine the copper contents in both carbon materials.

\section{General Procedure of Copper on Carbon-Catalyzed Click of 1,2,3-Triazole From Azides and Alkynes}

Azide (0.751 mmol, 1.2 equivalent), alkyne derivative (0.622 mmol, 1 equivalent), and $\mathrm{CuI}$ (0.005 equivalent) on carbon catalyst were placed in a reaction tube and $5 \mathrm{~mL}$ of water was added. The mixture was stirred for $6 \mathrm{~h}$ at room temperature. After completion of the reaction as evidenced by TLC, the 1,2,3-triazol product was extracted using diethyl ether and the $\mathrm{Cu}$ on carbon catalyst separated by filtration. The combined diethyl ether washings were evaporated under reduced pressure to afford the corresponding final pure 1,2,3-triazole. The recovered catalyst was dried and reused at least 10 times without any noticeable loss of its activity.

\section{Synthesis of}

\section{1-Benzyl-4-Phenyl-1H-1,2,3-Triazole (3a)}

White solid. Yield: $94 \% . R_{f}=0.3$ in hexane/ethyl acetate $(3: 1 \mathrm{v} / \mathrm{v})$. $\mathrm{Mp}=130-132{ }^{\circ} \mathrm{C} .{ }^{1} \mathrm{H}$ NMR $\left(400 \mathrm{MHz}, \mathrm{CDCl}_{3}, \delta \mathrm{ppm}\right): 5.60$ (s, $\left.2 \mathrm{H}, \mathrm{CH}_{2}\right) ; 7.28-7.44\left(\mathrm{~m}, 8 \mathrm{H}, \mathrm{CH}_{\mathrm{ar}}\right) ; 7.68\left(\mathrm{~s}, 1 \mathrm{H}, \mathrm{CH}_{\text {triazole }}\right) ; 7.81-$ $7.83\left(\mathrm{~d}, \mathrm{~J}=8.00 \mathrm{~Hz}, 2 \mathrm{H}, \mathrm{CH}_{\mathrm{ar}}\right) .{ }^{13} \mathrm{C} \mathrm{NMR}\left(100 \mathrm{MHz}, \mathrm{CDCl}_{3}, \delta\right.$ ppm): $54.4\left(\mathrm{CH}_{2}\right) ; 119.5\left(\mathrm{CH}_{\mathrm{ar}}\right) ; 125.9\left(3 \mathrm{CH}_{\mathrm{ar}}\right) ; 127.8\left(2 \mathrm{CH}_{\mathrm{ar}}\right)$; $128.2\left(2 \mathrm{CH}_{\mathrm{ar}}\right) ; 129.2\left(\mathrm{CH}_{\text {triazole }}\right) ; 131,5\left(\mathrm{C}_{\mathrm{ar}}\right) ; 135.0\left(\mathrm{C}_{\mathrm{ar}}\right) ; 148.3$ $\left(\mathrm{C}_{\text {triazole }}\right)$. HRMS $\left(\mathrm{FAB}^{+}\right) \mathrm{m} / z$ : Calcd for $\mathrm{C}_{15} \mathrm{H}_{14} \mathrm{~N}_{3}$ : 236.1188; Found: 236.1177. 


\section{Synthesis of Methyl 4-(1-Benzyl-1H-1,2,3-Triazol-4-yl)Benzoate} (3b)

White solid. Yield: $88 \% . R_{f}=0.42$ in hexane/ethyl acetate $(2: 1$ $\mathrm{v} / \mathrm{v}) . \mathrm{Mp}=176-178^{\circ} \mathrm{C} .{ }^{1} \mathrm{H} \mathrm{NMR}\left(400 \mathrm{MHz}, \mathrm{CDCl}_{3}, \delta \mathrm{ppm}\right): 3.91$ (s, $\left.3 \mathrm{H}, \mathrm{OCH}_{3}\right) ; 5.58\left(\mathrm{~s}, 2 \mathrm{H}, \mathrm{CH}_{2}\right) ; 7.33-7.38\left(\mathrm{~m}, 5 \mathrm{H}, \mathrm{CH}_{\mathrm{ar}}\right) ; 7.74$ $\left(\mathrm{s}, 1 \mathrm{H}, \mathrm{CH}_{\text {triazole }}\right) ; 7.86-7.89\left(\mathrm{~d}, J=12.00 \mathrm{~Hz}, 2 \mathrm{H}, \mathrm{CH}_{\mathrm{ar}}\right) ; 8.05-$ $8.08\left(\mathrm{~d}, J=12.00 \mathrm{~Hz}, 2 \mathrm{H}, \mathrm{CH}_{\mathrm{ar}}\right) .{ }^{13} \mathrm{C} \mathrm{NMR}\left(100 \mathrm{MHz}, \mathrm{CDCl}_{3}\right.$, $\delta$ ppm): $52.3\left(\mathrm{CH}_{3}\right) ; 54.5\left(\mathrm{CH}_{2}\right) ; 120.4\left(\mathrm{CH}_{\mathrm{ar}}\right) ; 125.6\left(2 \mathrm{CH}_{\mathrm{Ar}}\right)$; $128.3\left(2 \mathrm{CH}_{\mathrm{ar}}\right) ; 129.1\left(2 \mathrm{CH}_{\mathrm{ar}}\right) ; 129.4\left(2 \mathrm{C}_{\mathrm{ar}}\right) ; 129.7\left(\mathrm{CH}_{\text {triazolic }}\right)$; $130.3\left(\mathrm{C}_{\mathrm{ar}}\right) ; 134.5\left(\mathrm{C}_{\text {triazolic }}\right) ; 134.9\left(\mathrm{C}_{\mathrm{ar}}\right) ; 147.3\left(\mathrm{C}_{\mathrm{ar}}\right) ; 166.9$ (CO). HRMS $\left(\mathrm{FAB}^{+}\right) \mathrm{m} / z$ : Calcd for $\mathrm{C}_{17} \mathrm{H}_{16} \mathrm{~N}_{3} \mathrm{O}_{2}$ : 294.1243; Found: 294.1245.

\section{Synthesis of 4-(1-Benzyl-1H-1,2,3-Triazol- 4-yl)-N,N-Dimethylbenzenamine (3c)}

Yellow solid. Yield: $89 \% . R_{f}=0.26$ in hexane/ethyl acetate $(2: 1$ $\mathrm{v} / \mathrm{v}) . \mathrm{Mp}=202-204^{\circ} \mathrm{C} .{ }^{1} \mathrm{H}$ NMR $\left(400 \mathrm{MHz}, \mathrm{CDCl}_{3}, \delta \mathrm{ppm}\right)$ : 2.97 (s, $\left.3 \mathrm{H}, \mathrm{CH}_{3}\right) ; 5.54\left(\mathrm{~s}, 2 \mathrm{H}, \mathrm{CH}_{2}\right) ; 6.72-6.75$ (d, $J=12.0 \mathrm{~Hz}$, $\left.2 \mathrm{H}, \mathrm{CH}_{\mathrm{ar}}\right) ; 7.26-7.38\left(\mathrm{~m}, 5 \mathrm{H}, \mathrm{CH}_{\mathrm{ar}}\right) ; 7.53$ (s, $\left.1 \mathrm{H}, \mathrm{CH}_{\text {triazole }}\right)$; $7.65-$ $7.68\left(\mathrm{~d}, J=12.00 \mathrm{~Hz}, 2 \mathrm{H}, \mathrm{CH}_{\mathrm{ar}}\right) .{ }^{13} \mathrm{C} \mathrm{NMR}\left(100 \mathrm{MHz}, \mathrm{CDCl}_{3}\right.$, $\delta$ ppm): $40.6\left(\mathrm{CH}_{2}\right) ; 54.2\left(\mathrm{CH}_{3}\right) ; 112.5\left(2 \mathrm{CH}_{\mathrm{ar}}\right) ; 118.1\left(\mathrm{C}_{\mathrm{ar}}\right)$; $126.8\left(\mathrm{CH}_{\mathrm{ar}}\right) ; 128.1\left(2 \mathrm{CH}_{\mathrm{ar}}\right) ; 128.8\left(2 \mathrm{CH}_{\mathrm{ar}}\right) ; 129.2\left(2 \mathrm{CH}_{\mathrm{ar}}\right) ; 135.1$ $\left(\mathrm{C}_{\mathrm{ar}}+\mathrm{CH}_{\text {triazolic }}\right) ; 150.5\left(\mathrm{C}_{\text {triazolic }}\right)$. HRMS $\left(\mathrm{FAB}^{+}\right) \mathrm{m} / z$ : Calcd for $\mathrm{C}_{17} \mathrm{H}_{19} \mathrm{~N}_{4}$ : 279.1609; Found: 279.1599.

\section{Synthesis of 4-(1-Benzyl-1H-1,2,3-Triazol- 4-yl)Benzenamine (3d)}

White solid. Yield: $92 \% . R_{f}=0.48$ in hexane/ethyl acetate $(1: 2$ $\mathrm{v} / \mathrm{v}) . \mathrm{M}_{\mathrm{P}}=184^{\circ} \mathrm{C} .{ }^{1} \mathrm{H}$ NMR $\left(400 \mathrm{MHz}, \mathrm{CDCl}_{3}, \delta \mathrm{ppm}\right): 4.85$ (s, $\left.2 \mathrm{H}, \mathrm{NH}_{2}\right) ; 5.56\left(\mathrm{~s}, 2 \mathrm{H}, \mathrm{CH}_{2}\right) ; 6.72-6.75(\mathrm{~d}, J=12.00 \mathrm{~Hz}$, $\left.2 \mathrm{H}, \mathrm{CH}_{\mathrm{ar}}\right) ; 7.31-7.35\left(\mathrm{~m}, 5 \mathrm{H}, \mathrm{CH}_{\mathrm{ar}}\right) ; 7.49-7.52(\mathrm{~d}, J=12.00 \mathrm{~Hz}$, $\left.2 \mathrm{H}, \mathrm{CH}_{\mathrm{ar}}\right) ; 8.05$ (s, $\left.1 \mathrm{H}, \mathrm{CH}_{\text {triazole }}\right) .{ }^{13} \mathrm{C} \mathrm{NMR}\left(100 \mathrm{MHz}, \mathrm{CDCl}_{3}\right.$, $\delta \mathrm{ppm}): 55.36\left(\mathrm{CH}_{2}\right) ; 116.83\left(2 \mathrm{CH}_{\mathrm{ar}}\right) ; 121.07\left(\mathrm{C}_{\mathrm{ar}}\right) ; 121.44$ $\left(\mathrm{CH}_{\mathrm{ar}}\right) ; 128.17\left(2 \mathrm{CH}_{\mathrm{ar}}\right) ; 129.43\left(2 \mathrm{CH}_{\mathrm{ar}}\right) ; 129.96\left(2 \mathrm{CH}_{\mathrm{ar}}\right) ; 130.44$ $\left(\mathrm{CH}_{\text {triazolic }}\right) ; 137.32\left(\mathrm{C}_{\mathrm{ar}}\right) ; 149.90\left(\mathrm{C}_{\text {triazolic }}\right) ; 150.36\left(\mathrm{C}_{\mathrm{ar}}\right)$. HRMS $\left(\mathrm{FAB}^{+}\right) \mathrm{m} / z$ : Calcd for $\mathrm{C}_{15} \mathrm{H}_{15} \mathrm{~N}_{4}$ : 251.1297; Found: 251.1299 .

\section{Synthesis of Ethyl 1-Benzyl-1H-1,2,3- Triazole-4-Carboxylate (3e)}

White solid. Yield: $95 \% . R_{f}=0.29$ in hexane/ethyl acetate $(2: 1$ $\mathrm{v} / \mathrm{v}) . \mathrm{M}_{\mathrm{p}}=83-85^{\circ} \mathrm{C} .{ }^{1} \mathrm{H}$ NMR (400 MHz, $\left.\mathrm{CDCl}_{3}, \delta \mathrm{ppm}\right): 1.35-$ $1.40\left(\mathrm{t}, J=10.00 \mathrm{~Hz}, 3 \mathrm{H}, \mathrm{CH}_{3}\right) ; 4.35-4.42(\mathrm{q}, J=9.33 \mathrm{~Hz}, 4 \mathrm{H}$, $\left.\mathrm{OCH}_{2}\right) ; 5.57\left(\mathrm{~s}, 2 \mathrm{H}, \mathrm{CH}_{2}\right) ; 7.26-7.29\left(\mathrm{~m}, 3 \mathrm{H}, \mathrm{CH}_{\mathrm{ar}}\right) ; 7.37-7.4(\mathrm{~m}$, $\left.3 \mathrm{H}, \mathrm{CH}_{\mathrm{ar}}\right) ; 7.96\left(\mathrm{~s}, 1 \mathrm{H}, \mathrm{CH}_{\text {triazole }}\right) .{ }^{13} \mathrm{C} \mathrm{NMR}\left(100 \mathrm{MHz}, \mathrm{CDCl}_{3}\right.$, $\delta$ ppm): $14.4\left(\mathrm{CH}_{3}\right) ; 54.6\left(\mathrm{CH}_{2}\right) ; 61.4\left(\mathrm{CH}_{2}\right) ; 127.4\left(\mathrm{CH}_{\mathrm{ar}}\right)$; $128.4\left(2 \mathrm{CH}_{\mathrm{ar}}\right) ; 129.3\left(\mathrm{CH}_{\mathrm{ar}}\right) ; 129.4\left(\mathrm{CH}_{\mathrm{ar}}\right) ; 133.8\left(\mathrm{CH}_{\text {triazolic }}\right)$; $140.7\left(\mathrm{C}_{\text {triazolic }}\right) ; 160.8(\mathrm{CO})$. HRMS $\left(\mathrm{FAB}^{+}\right) \mathrm{m} / z$ : Calcd for $\mathrm{C}_{12} \mathrm{H}_{14} \mathrm{~N}_{3} \mathrm{O}_{2}$ : 232.1086; Found: 232.1087.

\section{Synthesis of}

\section{1,4-Diphenyl-1H-1,2,3-Triazole (3f)}

White solid. Yield: $89 \%$. $R_{f}=0.48$ in hexane/ethyl acetate $(3: 1$ $\mathrm{v} / \mathrm{v}) . \mathrm{M}_{\mathrm{P}}=183-184^{\circ} \mathrm{C} .{ }^{1} \mathrm{H}$ NMR $\left(400 \mathrm{MHz}, \mathrm{CDCl}_{3}, \delta \mathrm{ppm}\right)$ : 7.33-7.4 (m, 2H, $\left.\mathrm{CH}_{\mathrm{ar}}\right) ; 7.44-7.49\left(\mathrm{t}, J=7.47 \mathrm{~Hz}, 2 \mathrm{H}, \mathrm{CH}_{\mathrm{ar}}\right)$; $7.53-7.58\left(\mathrm{t}, J=7.56 \mathrm{~Hz}, 2 \mathrm{H}, \mathrm{CH}_{\mathrm{ar}}\right) ; 7.79-7.81(\mathrm{~d}, J=7.80 \mathrm{~Hz}$, $\left.2 \mathrm{H}, \mathrm{CH}_{\mathrm{ar}}\right) ; 7.92-7.94\left(\mathrm{~d}, J=7.93 \mathrm{~Hz}, 2 \mathrm{H}, \mathrm{CH}_{\mathrm{ar}}\right) ; 8.22(\mathrm{~s}, 1 \mathrm{H}$, $\left.\mathrm{CH}_{\text {triazole }}\right) .{ }^{13} \mathrm{C} \mathrm{NMR}\left(100 \mathrm{MHz}, \mathrm{CDCl}_{3}, \delta \mathrm{ppm}\right): 118.07\left(2 \mathrm{CH}_{\mathrm{ar}}\right)$; $120.98\left(\mathrm{C}_{\mathrm{ar}}\right)$; $126.34\left(2 \mathrm{CH}_{\mathrm{ar}}\right) ; 128,98\left(\mathrm{CH}_{\mathrm{ar}}\right) ; 129,31\left(\mathrm{CH}_{\mathrm{ar}}\right)$; $129,37\left(2 \mathrm{CH}_{\mathrm{ar}}\right) ; 130,23\left(2 \mathrm{CH}_{\mathrm{ar}}\right) ; 130.34\left(\mathrm{CH}_{\text {triazolic }}\right) ; 130.73\left(\mathrm{C}_{\mathrm{ar}}\right)$; $137.47\left(\mathrm{C}_{\text {triazolic }}\right)$. HRMS $\left(\mathrm{FAB}^{+}\right) \mathrm{m} / z$ : Calcd for $\mathrm{C}_{14} \mathrm{H}_{12} \mathrm{~N}_{3}$ : 222.1031; Found: 222.1029.

\section{Synthesis of N,N-Dimethyl-4-(1-Phenyl-1H- 1,2,3-Triazol-4-yl)Benzenamine (3g)}

Yellow solid. Yield: $87 \%$. $R_{f}=0.5$ in hexane/ethyl acetate $(2: 1$ $\mathrm{v} / \mathrm{v}) . \mathrm{M}_{\mathrm{p}}=169-171^{\circ} \mathrm{C} .{ }^{1} \mathrm{H}$ NMR $\left(400 \mathrm{MHz}, \mathrm{CDCl}_{3}, \delta \mathrm{ppm}\right)$ : $2.19\left(\mathrm{~s}, 1 \mathrm{H}, \mathrm{CH}_{3}\right) ; 6.81-6.84\left(\mathrm{~d}, J=12.00 \mathrm{~Hz}, 2 \mathrm{H}, \mathrm{CH}_{\mathrm{ar}}\right) ; 7.43-$ $7.58\left(\mathrm{~m}, 5 \mathrm{H}, \mathrm{CH}_{\mathrm{ar}}\right) ; 7.80-7.82\left(\mathrm{~d}, J=8.00 \mathrm{~Hz}, 2 \mathrm{H}, \mathrm{CH}_{\mathrm{ar}}\right) ; 8.08$ (s, $\left.1 \mathrm{H}, \mathrm{CH}_{\text {triazole }}\right) .{ }^{13} \mathrm{C} \mathrm{NMR}\left(100 \mathrm{MHz}, \mathrm{CDCl}_{3}, \delta \mathrm{ppm}\right): 40.6$ $\left(2 \mathrm{CH}_{3}\right) ; 112.6\left(2 \mathrm{CH}_{\mathrm{ar}}\right) ; 116.1\left(2 \mathrm{CH}_{\mathrm{ar}}\right) ; 120.6\left(5 \mathrm{CH}_{\mathrm{ar}}\right) ; 126.9$ $\left(\mathrm{C}_{\mathrm{ar}}+\mathrm{CH}_{\text {triazolic }}\right) ; 130.1\left(\mathrm{C}_{\text {triazolic }}\right) ; 141\left(\mathrm{C}_{\mathrm{ar}}\right)$. HRMS $\left(\mathrm{FAB}^{+}\right) \mathrm{m} / z$ : Calcd for $\mathrm{C}_{16} \mathrm{H}_{17} \mathrm{~N}_{4}$ : 265.1453; Found: 265.1444.

\section{Synthesis of Ethyl 1-Phenyl-1H-1,2,3- Triazole-4-Carboxylate (3h)}

White solid. Yield: $73 \% . R_{f}=0.56$ in hexane/ethyl acetate $(2: 1$ $\mathrm{v} / \mathrm{v}) . \mathrm{M}_{\mathrm{p}}=75-77^{\circ} \mathrm{C} .{ }^{1} \mathrm{H} \mathrm{NMR}\left(400 \mathrm{MHz}, \mathrm{CDCl}_{3}, \delta \mathrm{ppm}\right): 1.41-$ $1.45\left(\mathrm{t}, J=8.00 \mathrm{~Hz}, 3 \mathrm{H}, \mathrm{CH}_{3}\right) ; 4.43-4.5(\mathrm{q}, J=9.33 \mathrm{~Hz}, 4 \mathrm{H}$, $\left.\mathrm{CH}_{2}\right) ; 7.46-7.58\left(\mathrm{~m}, 3 \mathrm{H}, \mathrm{CH}_{\mathrm{ar}}\right) ; 7.74-7.76\left(\mathrm{~m}, 3 \mathrm{H}, \mathrm{CH}_{\mathrm{ar}}\right) ; 8.51$ (s, $\left.1 \mathrm{H}, \mathrm{CH}_{\text {triazole }}\right) .{ }^{13} \mathrm{C} \mathrm{NMR}\left(100 \mathrm{MHz}, \mathrm{CDCl}_{3}, \delta \mathrm{ppm}\right): 31.1$ $\left(\mathrm{CH}_{3}\right) ; 61.6\left(\mathrm{CH}_{2}\right) ; 120.9\left(\mathrm{C}_{\mathrm{ar}}\right) ; 125.6\left(2 \mathrm{CH}_{\mathrm{ar}}\right) ; 129.6\left(2 \mathrm{CH}_{\mathrm{ar}}\right)$; $130.1\left(\mathrm{CH}_{\text {triazolic }}\right) ; 136.5\left(\mathrm{C}_{\text {triazolic }}\right) ; 141(\mathrm{CO})$. HRMS $\left(\mathrm{FAB}^{+}\right)$ $m / z$ : Calcd for $\mathrm{C}_{11} \mathrm{H}_{12} \mathrm{~N}_{3} \mathrm{O}_{2}$ : 218.0929; Found: 218.0924 .

\section{Synthesis of 1-(4-Methoxybenzyl)-4- Phenyl-1H-1,2,3-Triazole (3i)}

White solid. Yield: $96 \% . R_{f}=0.28$ in hexane/ethyl acetate $(3: 1$ $\mathrm{v} / \mathrm{v}) \cdot \mathrm{M}_{\mathrm{p}}=132-135^{\circ} \mathrm{C} .{ }^{1} \mathrm{H} \mathrm{NMR}\left(400 \mathrm{MHz}, \mathrm{CDCl}_{3}, \delta \mathrm{ppm}\right): 3.73$ $\left(\mathrm{s}, 3 \mathrm{H}, \mathrm{OCH}_{3}\right) ; 5.43\left(\mathrm{~s}, 2 \mathrm{H}, \mathrm{CH}_{2}\right) ; 6.82-6.85(\mathrm{~d}, J=12.00 \mathrm{~Hz}, 2 \mathrm{H}$, $\left.\mathrm{CH}_{\mathrm{ar}}\right) ; 7.18-7.82\left(\mathrm{~m}, 5 \mathrm{H}, \mathrm{CH}_{\mathrm{ar}}\right) ; 7.54\left(\mathrm{~s}, 1 \mathrm{H}, \mathrm{CH}_{\text {triazole }}\right) ; 7.69-7.72$ $\left(\mathrm{d}, J=12.00 \mathrm{~Hz}, 2 \mathrm{H}, \mathrm{CH}_{\mathrm{ar}}\right) .{ }^{13} \mathrm{C} \mathrm{NMR}\left(100 \mathrm{MHz}, \mathrm{CDCl}_{3}, \delta \mathrm{ppm}\right)$ $53.9\left(\mathrm{CH}_{2}\right) ; 55.4\left(\mathrm{CH}_{3}\right) ; 114.6\left(2 \mathrm{CH}_{\mathrm{ar}}\right) ; 119.3\left(2 \mathrm{CH}_{\mathrm{ar}}\right) ; 125.8$ $\left(2 \mathrm{CH}_{\mathrm{ar}}\right) ; 128.2\left(\mathrm{CH}_{\mathrm{ar}}\right) ; 128.9\left(2 \mathrm{CH}_{\mathrm{ar}}\right) ; 129.8\left(\mathrm{C}_{\mathrm{ar}}\right) ; 128.8\left(2 \mathrm{CH}_{\mathrm{ar}}\right)$; $130.7\left(\mathrm{C}_{\mathrm{ar}}+\mathrm{CH}_{\text {triazolic }}\right) ; 148.2\left(\mathrm{C}_{\text {triazolic }}\right) ; 160.1\left(\mathrm{CH}_{\mathrm{ar}}\right)$. HRMS $\left(\mathrm{FAB}^{+}\right) \mathrm{m} / z$ : Calcd for $\mathrm{C}_{16} \mathrm{H}_{16} \mathrm{~N}_{3} \mathrm{O}$ : 266.1293; Found: 266.1286 .

\section{Synthesis of Methyl 4-(1-(4-Methoxybenzyl)-1H-1,2,3-Triazol-4- yl)Benzoate (3j)}

White solid. Yield: $80 \% . R_{f}=0.66$ in hexane/ethyl acetate $(1: 1$ $\mathrm{v} / \mathrm{v}) . \mathrm{M}_{\mathrm{p}}=183-185^{\circ} \mathrm{C} .{ }^{1} \mathrm{H} \mathrm{NMR}\left(400 \mathrm{MHz}, \mathrm{CDCl}_{3}, \delta \mathrm{ppm}\right)$ : $3.74\left(\mathrm{~s}, 3 \mathrm{H}, \mathrm{OCH}_{3}\right) ; 3.84\left(\mathrm{~s}, 3 \mathrm{H}, \mathrm{OCH}_{3}\right) ; 5.44\left(\mathrm{~s}, 2 \mathrm{H}, \mathrm{CH}_{2}\right) ; 6.83-$ $6.86\left(\mathrm{~d}, J=12.00 \mathrm{~Hz}, 2 \mathrm{H}, \mathrm{CH}_{\mathrm{ar}}\right) ; 7.19-7.22(\mathrm{~d}, J=12.00 \mathrm{~Hz}$, $\left.2 \mathrm{H}, \mathrm{CH}_{\mathrm{ar}}\right) ; 7.63\left(\mathrm{~s}, 1 \mathrm{H}, \mathrm{CH}_{\text {triazole }}\right) ; 7.77-7.80(\mathrm{~d}, J=12.00 \mathrm{~Hz}$, $\left.2 \mathrm{H}, \mathrm{CH}_{\mathrm{ar}}\right) ; 7.97-8.00\left(\mathrm{~d}, \mathrm{~J}=12.00 \mathrm{~Hz}, 2 \mathrm{H}, \mathrm{CH}_{\mathrm{ar}}\right) .{ }^{13} \mathrm{C} \mathrm{NMR}$ (100 MHz, $\left.\mathrm{CDCl}_{3}, \delta \mathrm{ppm}\right): 52.1\left(\mathrm{CH}_{3}\right) ; 53.9\left(\mathrm{CH}_{2}\right) ; 55.3\left(\mathrm{CH}_{3}\right)$; $114.5\left(2 \mathrm{CH}_{\mathrm{ar}}\right) ; 125.4\left(2 \mathrm{CH}_{\mathrm{ar}}\right) ; 129.5\left(2 \mathrm{CH}_{\mathrm{ar}}\right) ; 129.7\left(2 \mathrm{C}_{\mathrm{ar}}\right) ; 130.1$ $\left(\mathrm{CH}_{\text {triazolic }}\right) ; 134.9\left(\mathrm{C}_{\text {triazolic }}\right) ; 147.1\left(\mathrm{C}_{\mathrm{ar}}\right) ; 160.0\left(\mathrm{C}_{\mathrm{ar}}\right) ; 166.7$ (CO). HRMS $\left(\mathrm{FAB}^{+}\right) \mathrm{m} / z$ : Calcd for $\mathrm{C}_{18} \mathrm{H}_{18} \mathrm{~N}_{3} \mathrm{O}_{3}$ : 324.1348; Found: 324.1338. 
TABLE 1 | The specific surface area, average pore diameter, and $V_{\text {total }}$ of pores of CANS and CC.

\begin{tabular}{|c|c|c|c|}
\hline $\begin{array}{l}\text { Activated } \\
\text { carbon }\end{array}$ & $\mathrm{S}_{\mathrm{BET}}\left(\mathrm{m}^{2} / \mathrm{g}\right)$ & $\begin{array}{l}\text { Average pore } \\
\text { diameter }(\mathrm{nm})\end{array}$ & $\begin{array}{c}\mathbf{V}_{\text {Total }} \text { of pores } \\
\left(\mathrm{cm}^{3} / \mathrm{g}\right)\end{array}$ \\
\hline CANS & 1151.75 & 2.204 & 0.635 \\
\hline $\mathrm{CC}$ & 702.76 & 1.168 & 0.193 \\
\hline
\end{tabular}

CANS, carbon from Argan Nut Shells biomass; CC, commercially carbon.

\section{Synthesis of 4-(1-(4-Methoxybenzyl)-1H- 1,2,3-Triazol-4-yl)Benzenamine (3k)}

Yellow solid. Yield: $78 \% . R_{f}=0.34$ in hexane/ethyl acetate $(1: 1$ $\mathrm{v} / \mathrm{v}) . \mathrm{M}_{\mathrm{p}}=121-123^{\circ} \mathrm{C} .{ }^{1} \mathrm{H}$ NMR $\left(400 \mathrm{MHz}, \mathrm{CDCl}_{3}, \delta \mathrm{ppm}\right)$ : 2.09 (s, $\left.1 \mathrm{H}, \mathrm{NH}_{2}\right) ; 3.73$ (s, 3H, $\left.\mathrm{OCH}_{3}\right) ; 5.40\left(\mathrm{~s}, 2 \mathrm{H}, \mathrm{CH}_{2}\right) ; 6.61-$ $6.64\left(\mathrm{~d}, J=12.00 \mathrm{~Hz}, 2 \mathrm{H}, \mathrm{CH}_{\mathrm{ar}}\right) ; 6.81-6.84(\mathrm{~d}, J=12.00 \mathrm{~Hz}$, $\left.2 \mathrm{H}, \mathrm{CH}_{\mathrm{ar}}\right) ; 7.17-7.19\left(\mathrm{~d}, J=8.00 \mathrm{~Hz}, 2 \mathrm{H}, \mathrm{CH}_{\mathrm{ar}}\right) ; 7.41(\mathrm{~s}, 1 \mathrm{H}$, $\left.\mathrm{CH}_{\text {triazole }}\right) ; 7.49-7.52\left(\mathrm{~d}, \mathrm{~J}=12.00 \mathrm{~Hz}, 2 \mathrm{H}, \mathrm{CH}_{\mathrm{ar}}\right) .{ }^{13} \mathrm{C} \mathrm{NMR}(100$ $\left.\mathrm{MHz}, \mathrm{CDCl}_{3}, \delta \mathrm{ppm}\right): 53.6\left(\mathrm{CH}_{3}\right) ; 55.3\left(\mathrm{CH}_{2}\right) ; 114.4\left(2 \mathrm{CH}_{\mathrm{ar}}\right)$; $115.1\left(2 \mathrm{CH}_{\mathrm{ar}}\right) ; 117.9\left(2 \mathrm{CH}_{\mathrm{ar}}\right) ; 126.8\left(2 \mathrm{CH}_{\mathrm{ar}}\right) ; 126.9\left(2 \mathrm{C}_{\mathrm{ar}}\right) ; 129.6$ $\left(\mathrm{C}_{\mathrm{ar}}+\mathrm{CH}_{\text {triazolic }}\right) ; 146.4\left(\mathrm{C}_{\text {triazolic }}\right) ; 159.8\left(\mathrm{C}_{\mathrm{ar}}\right)$. HRMS $\left(\mathrm{FAB}^{+}\right)$ $m / z$ : Calcd for $\mathrm{C}_{16} \mathrm{H}_{17} \mathrm{~N}_{4} \mathrm{O}$ : 381.1402; Found: 281.1398 .

\section{RESULTS AND DISCUSSION}

\section{Synthesis and Characterization of Copper on Carbon Catalyst}

The synthesis of activated carbon from the local biomass such as Argan nut shells by the chemical activation method was adopted in this study. The advantage of this activation is to opt for low pyrolysis temperatures and a smaller activation cost. The high quality of activated carbon with a very large porous texture and high surface area was prepared from Argan nut shells biomass by using orthophosphoric acid as activating agent. This activation process aims to develop and modulate the porous structure of carbon, leading to a very sharp increase in its specific surface area. Indeed, the specific surface area of activated carbon is one of the most important physical structure parameters, the accuracy of its measured value being essential for realistic reference significance. In this work, the dinitrogen adsorption method was adopted to measure the specific surface area of activated carbon and the results obtained are summarized in Table 1. In this work, the Brunauer-Emmett-Teller (BET) method was adopted to measure the specific surface area of activated carbon and the corresponding results are also summarized in Table 1. It can be deduced from such results that commercially available carbon material CC, used for comparative purposes, presents a specific surface area equal to $\mathrm{S}_{\mathrm{BET}}=702.76 \mathrm{~m}^{2} / \mathrm{g}$ with microspores rounding a size $<2 \mathrm{~nm}$, while the prepared carbon material CANS exhibits a highly porous structure as shown by the high found value of the specific surface area found to be $S_{\mathrm{BET}}=$ $1151.75 \mathrm{~m}^{2} / \mathrm{g}$ ) and the mesoporous character evaluated at $2.2 \mathrm{~nm}$. This behavior allows easy access and contact between the reagents employed in a given heterogeneous catalytic protocol.

The $\mathrm{Cu}$-carbon catalysts were prepared by impregnation of carbon supports with $\mathrm{CuI}$ in acetonitrile overnight at

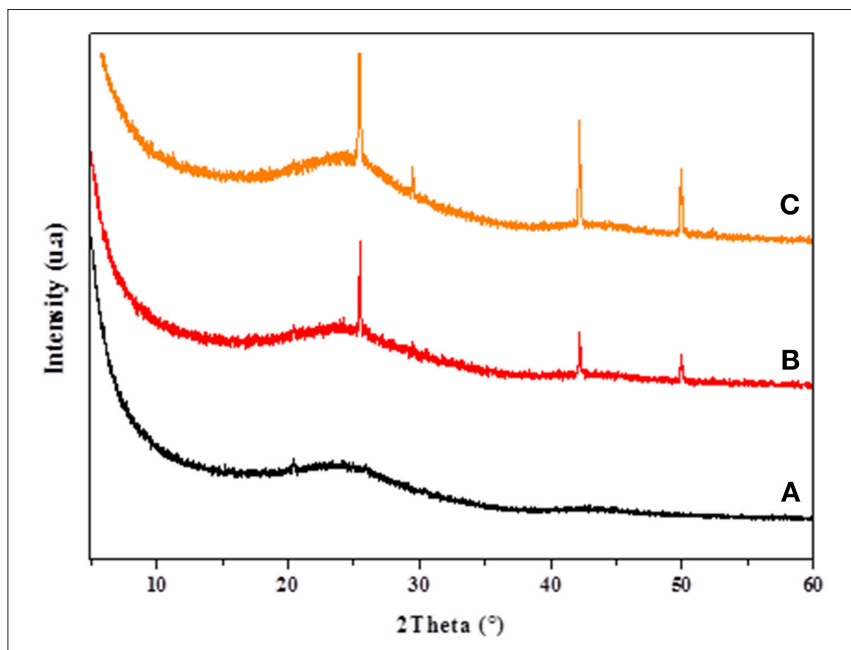

FIGURE 1 | XRD results of CANS support (A), Cu-CANS material (B), and phenylacetylene in Cu-CANS (C).

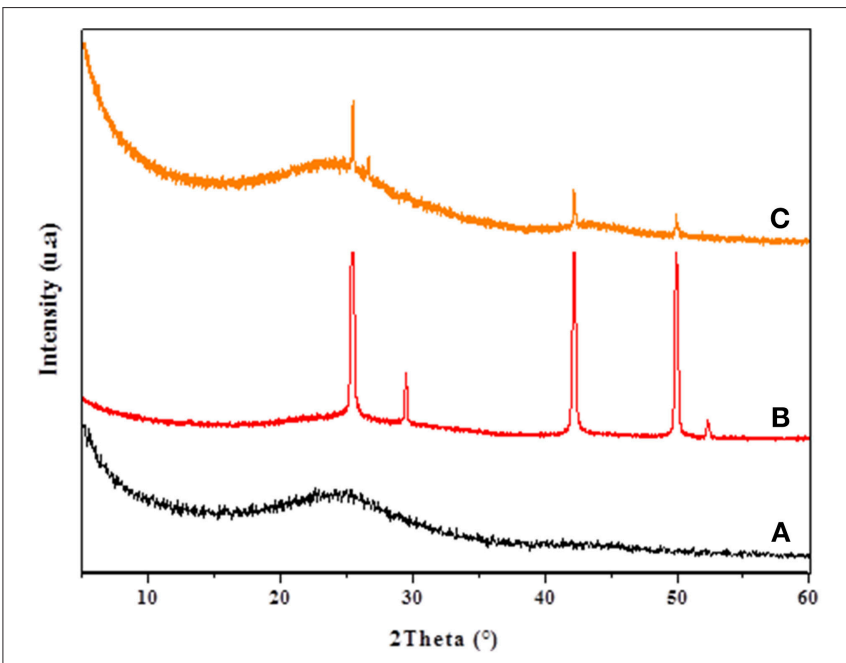

FIGURE 2 | XRD results of CC support (A), Cu-CC material (B), and phenylacetylene in $\mathrm{Cu}-\mathrm{CC}$ (C)

room temperature. The reaction mixture filtered, and the solid successively washed with acetonitrile, diethyl ether and dried under vacuum. The catalysts were characterized by several techniques such as Scanning electron microscopy (SEM), Energydispersive X-ray (EDX), X-ray diffraction (XRD) analysis, and FT-IR spectroscopy.

The XRD patterns of $\mathrm{CC}, \mathrm{CANS}, \mathrm{Cu}-\mathrm{CC}$, and $\mathrm{Cu}-\mathrm{CANS}$ are presented in Figures 1, 2. The obtained XRD patterns displayed the following diffraction peaks $\left(2 \theta\left[{ }^{\circ}\right]\right): 25.5,29.46,42.19$, and $49.91^{\circ}$ for $\mathrm{Cu}-\mathrm{CANS}$, (Figure 1) which can be correlated to the (111), (200), (220), and (311) hkl indices, respectively, of CuI (JCPDS no. 06-0246). As shown in Figure 2, five peaks at $2 \theta$ $=25.47,29.46,42.34,49.96$, and $52.30^{\circ}$ corresponding to the (111), (200), (220), (311), and (222) planes of CuI (JCPDS no. 06-0246) were observed in the pattern of the $\mathrm{Cu}$-CC composite, 


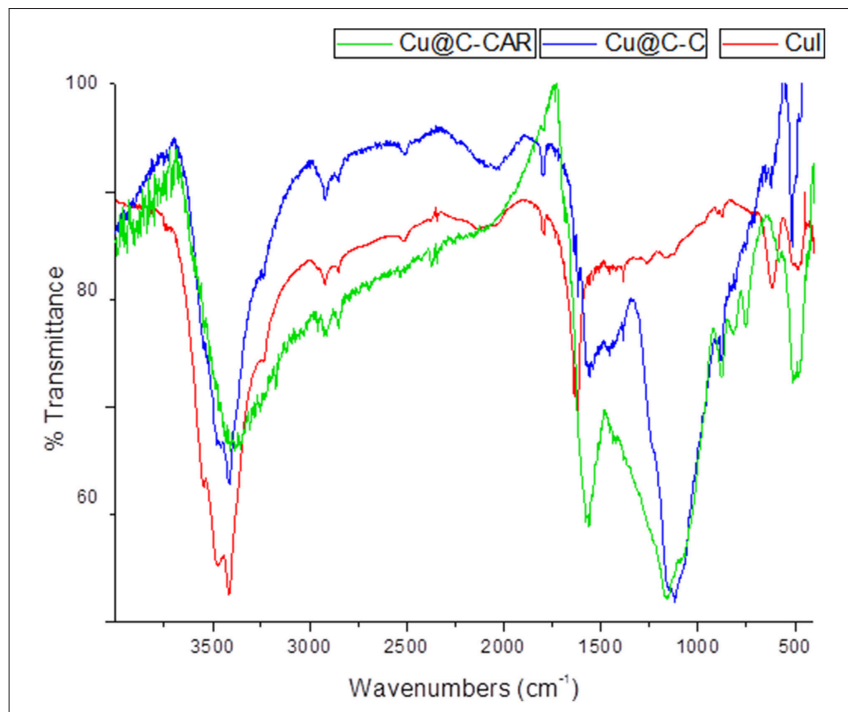

FIGURE 3 | Comparative FT-IR spectra of Cul precursor (red), Cu-CC (blue), and Cu-CANS material (green).

indicating that $\mathrm{CuI}$ has been successfully loaded on the carbons. The average crystallite size $D$ of the nanoparticles is calculated from the Scherrer equation: $D=K \lambda /(\beta \cos \theta)$, where $K$ is the Debye-Scherrer constant (0.9), $\lambda$ is the X-ray wavelength, $\beta$ FWHM (full-width at half-maximum or half width) is in radians, and $\theta$ is the Bragg diffraction angle. Here, the (111) peak of the highest intensity was picked out to evaluate the particle diameter of CuI. The values of the $D$ constants were calculated to be about 18.81 and $26.15 \mathrm{~nm}$ for $\mathrm{nm}$ for $\mathrm{Cu}-\mathrm{CC}$ and Cu-CANS, respectively.

Infrared spectroscopy is one of the most widely used techniques in heterogeneous catalysis to characterize and identify the purity of solids by the presence of characteristic bands of extraneous compounds. Comparison between FT-IR spectrum of copper particles on activated carbon with that of copper iodide and with the carbon support reveals the appearance of weak absorption bands at $496 \mathrm{~cm}^{-1}$ (Figure 3). This is related to the supported copper particles on activated carbon. Furthermore, the change observed in the absorption peaks of the carbonyl group from 1654.7 to 1650.4 and 1561.4 to $1557 \mathrm{~cm}^{-1}$ is related to the coordination of oxygen atoms (acetate and phosphate) to copper metal ions (Samim et al., 2007; Ghouma et al., 2017). The presence of oxygen atoms (phosphate-containing groups) may afford the assembly of polynuclear copper ions in the carbon support because of the known bridging ability of the phosphate groups (Ikotun et al., 2010).

We used the scanning electron microscopy (SEM) to study and visualize the morphology of the surface before and after the immobilization of copper on activated carbons. The SEM images of activated carbons and catalysts clearly reveal the presence of different size pores on the raw activated carbons and showed that both catalysts have the same shape and contain a mosaic of copper particles of different sizes and morphologies (Figure 4). The energy dispersive X-ray

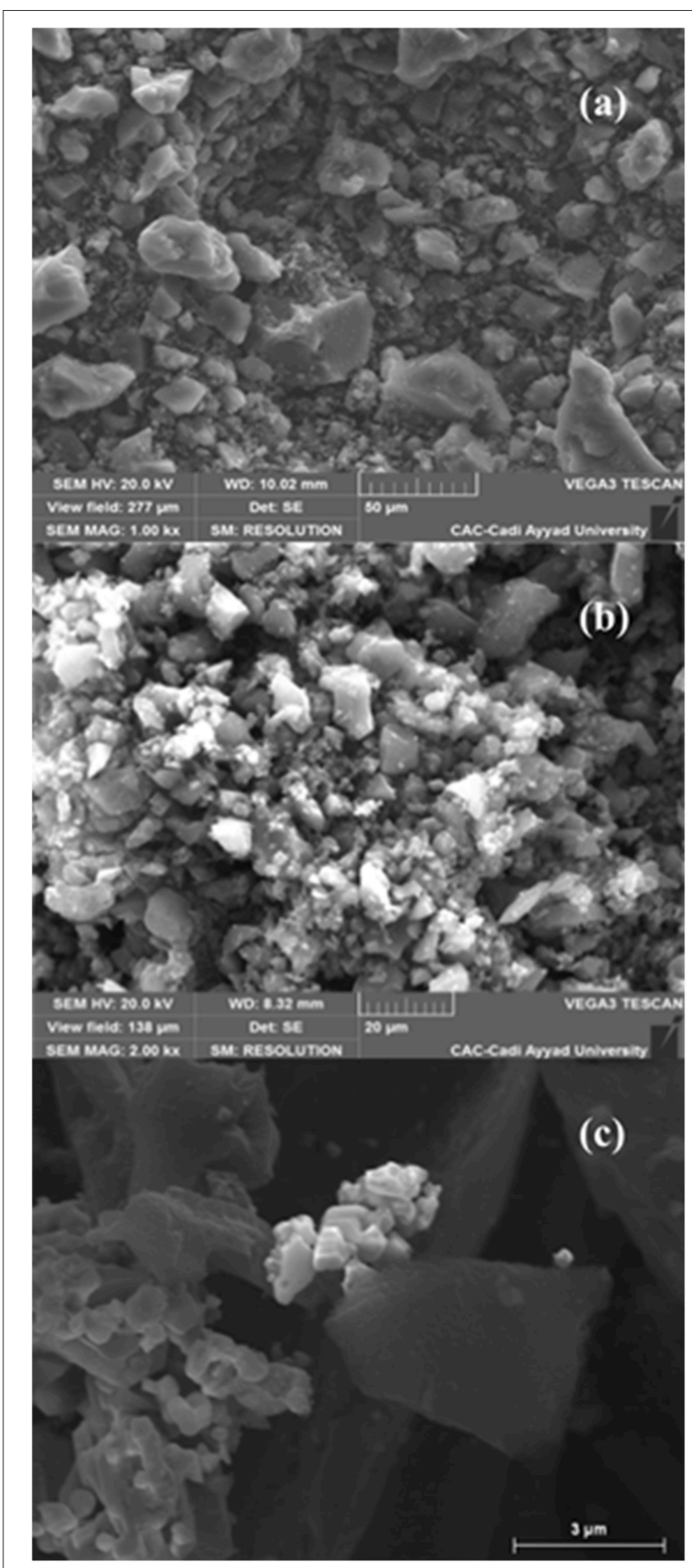

FIGURE 4 | SEM images of CANS support (a), Cu-CANS (b), and Cu-CC material (c).

(EDX) results obtained from the SEM analysis for the CANS, and $\mathrm{Cu}$-CANS showed the presence of $\mathrm{C}, \mathrm{O}, \mathrm{Al}$ and $\mathrm{P}$ atoms for the former and $\mathrm{C}, \mathrm{Cu}$, and $\mathrm{P}$ atoms for the latter (Figure 5). 


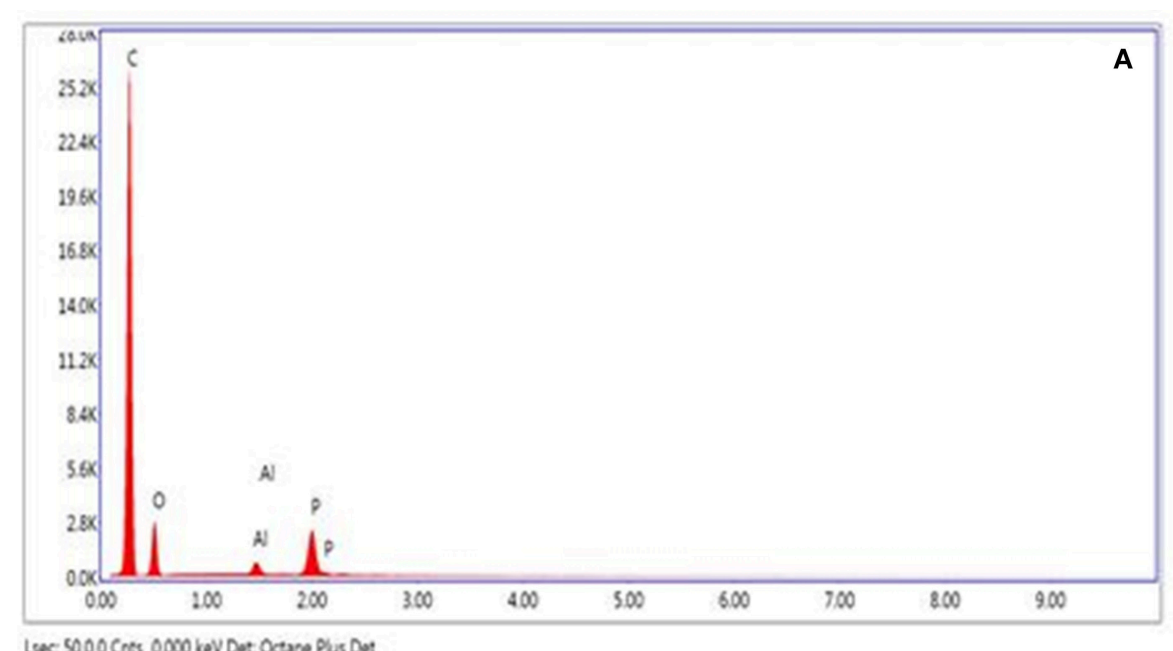

Lec: $\$ 000$ Cns 0000 keV Det Octane Pus Det

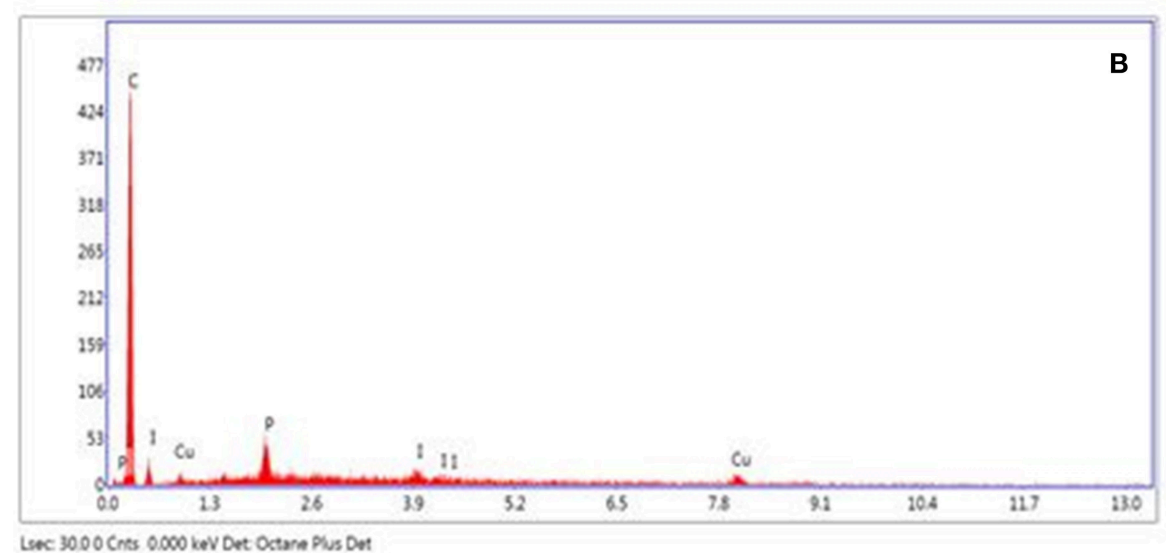

FIGURE 5 | EDX spectrum of (A) CANS and (B) Cu-CANS catalyst.

The copper contents in the carbon materials were determined by AAS analysis and found to be $1.38 \mathrm{wt} \%$ of $\mathrm{Cu}$ for $\mathrm{Cu}-$ CANS and $3.82 \mathrm{wt} \%$ of $\mathrm{Cu}$ for $\mathrm{Cu}$-CC. Thus, $100 \mathrm{mg}$ of carbon composite contains $2.18 \mathrm{~mol} \%$ of copper for $\mathrm{Cu}$-CANS and $6.01 \mathrm{~mol} \%$ of copper for $\mathrm{Cu}-\mathrm{CC}$. The low copper loadings in $\mathrm{Cu}$-CANS compared with $\mathrm{Cu}-\mathrm{CC}$ can be explained by their respective preparation method and the functionalizing groups that may coordinate to the copper ions in the carbon material.

\section{Adsorption Isotherms}

The equilibrium adsorption isotherms are very important for understanding the mechanism of the $\mathrm{CuI}$ adsorption on both activated carbons investigated. The adsorption data were analyzed with the help of the following linear forms of Freundlich and Langmuir isotherms.

The Langmuir isotherm is valid for monolayer adsorption on surface containing a finite number of identical sites (Langmuir, 1916). The linear form of the Langmuir isotherm can be represented by the following equation:

$$
\frac{C_{e}}{q_{e}}=\frac{1}{\bar{q}_{m} K_{L}}+\frac{1}{q_{m}} C_{e}
$$

where $C_{e}(\mathrm{mg} / \mathrm{L})$ represents the equilibrium concentration of the adsorbate, $q_{e}$ is the amount adsorbed at equilibrium $(\mathrm{mg} / \mathrm{g})$, and $K_{L}(\mathrm{~L} / \mathrm{mg})$ and $q_{m}(\mathrm{mg} / \mathrm{g})$ stand for the Langmuir constant and the maximum amount of adsorbate, respectively.

The Freundlich isotherm model is an empirical equation based on sorption on a heterogeneous surface or surface supporting sites of varied affinities (Freundlich, 1906). The linearized Freundlich model is represented by the following equation:

$$
\log \left(q_{e}\right)=\log \left(K_{f}\right)+\frac{1}{n} \log \left(C_{e}\right)
$$

where $K_{f}(\mathrm{mg} / \mathrm{g})$ is the Freundlich constants related to the sorption capacity and $n$ is the heterogeneity factor.

As shown in Table 2, the comparison of the Freundlich and Langmuir models, reveals that the values of the correlation 
TABLE 2 | Isotherm constants for adsorption of Cul on CANS and CC activated carbons.

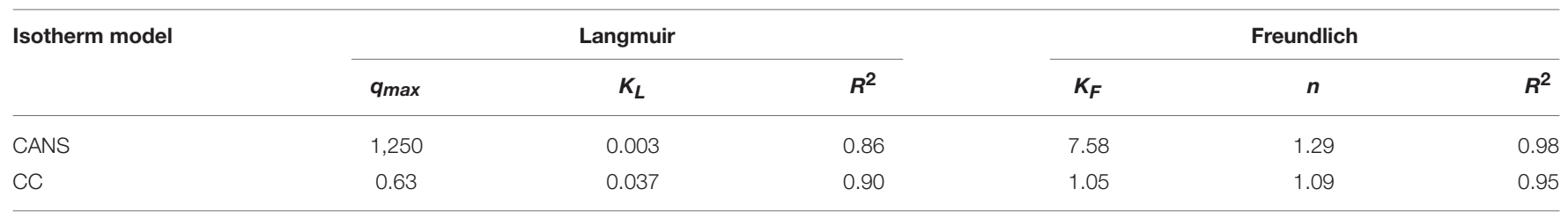

TABLE 3 | Catalyst and conditions screening for the cycloaddition of benzyl azide and phenylacetylene ${ }^{\mathrm{a}}$.<smiles>C#Cc1ccccc1</smiles>

alkyne (1a)<smiles>[N]Cc1ccccc1</smiles>

azide (2a)

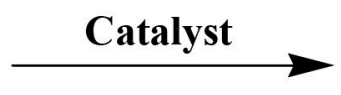

Solvent / r.t<smiles>c1ccc(Cn2cc(-c3ccccc3)nn2)cc1</smiles>

1,4-isomer

\begin{tabular}{|c|c|c|c|c|c|}
\hline Entry & Catalyst & Cat. loading (mol \%) & Solvent & Time (h) & Yield $(\%)^{b}$ \\
\hline 1 & - & - & Water & 24 & 0 \\
\hline 2 & $\mathrm{CC}$ & - & Water & 24 & 0 \\
\hline 3 & $\mathrm{CuCl}-\mathrm{CC}$ & 5 & Water & 6 & 66 \\
\hline 4 & $\mathrm{CuBr}-\mathrm{CC}$ & 5 & Water & 6 & 74 \\
\hline 5 & Cul-CC & 3 & Water & 6 & 99 \\
\hline 8 & Cul-CC & 0.1 & Water & 6 & 74 \\
\hline 9 & Cul-CC & 5 & Ethanol & 6 & 85 \\
\hline 10 & Cul-CC & 5 & Methanol & 6 & 74 \\
\hline 11 & Cul-CC & 5 & Toluene & 6 & 80 \\
\hline 12 & Cul-CC & 5 & Acetonitrile & 6 & 98 \\
\hline
\end{tabular}

${ }^{a}$ Reaction conditions: benzylazide $(0.75 \mathrm{mmol})$; phenylacetylene $(0.62 \mathrm{mmol})$; solvent $(5 \mathrm{~mL})$; and catalyst were mixed and stirred at room temperature. ${ }^{b}$ Isolated yields.

coefficient of the Freundlish isotherm $\left(R^{2}=0.98\right.$ and 0.95$)$ are high for both activated carbons. This result suggests that the CuI was heterogeneously adsorbed on a multilayer surface and the values found for $n$ were $>1$, a feature which proves that the adsorption on both adsorbents is favorable.

\section{Catalytic Activity of the Cu-Carbon Catalytic System in the Synthesis of 1,2,3-Triazole Derivatives}

In order to explore the catalytic activity of the synthesized catalysts and to optimize the reaction conditions for the $[3+2]$ cycloaddition, the reaction between benzyl azide and phenylacetylene was chosen as a model [3+2] cycloaddition reaction. As starting point, different reaction conditions such as copper sources, solvent, and amount of catalyst were investigated (see Table 3). The control experiments show that the $[3+2]$ cycloaddition reaction azide-alkyne does not take place in the absence of the catalyst (entries 1-3, Table 3). Moreover, the effect of copper(I) sources supported within the pores of both
CC and CANS was examined (entries 4-6, Table 3). In fact, the results shown in Table 3 confirm the recently established catalytic activity trend found in CuAAC assisted by several Nheterocylic carbene copper(I) complexes, namely [(NHC)CuX] $[\mathrm{NHC}=N$-Heterocycle carbene; $\mathrm{X}=\mathrm{I}, \mathrm{Cl}, \mathrm{Br}]:[\mathrm{CuI}(\mathrm{NHC})]$ $>[\mathrm{CuBr}(\mathrm{NHC})]>[\mathrm{CuCl}(\mathrm{NHC})]$ (Díez-Gonzalez et al., 2010). Our results show that among the studied $\mathrm{Cu}(\mathrm{I})$-carbon catalysts, CuI-CC and CuI-CANS are the most efficient ones for the 1,2,3triazole click reactions performed under strict click chemistry conditions, specifically by using water as solvent and working at room (entries 4-6, Table 3). Having identified the CuI-CC and CuI-CANS as efficient catalytic systems for the reactions; we then explored the effect of the catalyst amount on the conversion yields and the catalyst loading being enhanced from 0.1 to 3 $\mathrm{mol} \%$. As a result, the conversion yields were increased from 70 to $99 \%$ by the increasing of catalyst amount (entries 6-8, Table 3 ). However, no elevated conversion yields were observed once the catalyst amount becomes greater than $3 \mathrm{~mol} \%$. Consequently, in both $\mathrm{Cu}(\mathrm{I})-\mathrm{CC}$ and $\mathrm{Cu}(\mathrm{I})-\mathrm{CANS}$ catalysts, a catalyst loading of $0.5 \mathrm{~mol} \%$ appears to be optimal with respect to excellent yields 
TABLE 4 | Cycloaddition of azides and alkynes catalyzed by copper-carbon catalysts ${ }^{\mathrm{a}}$.

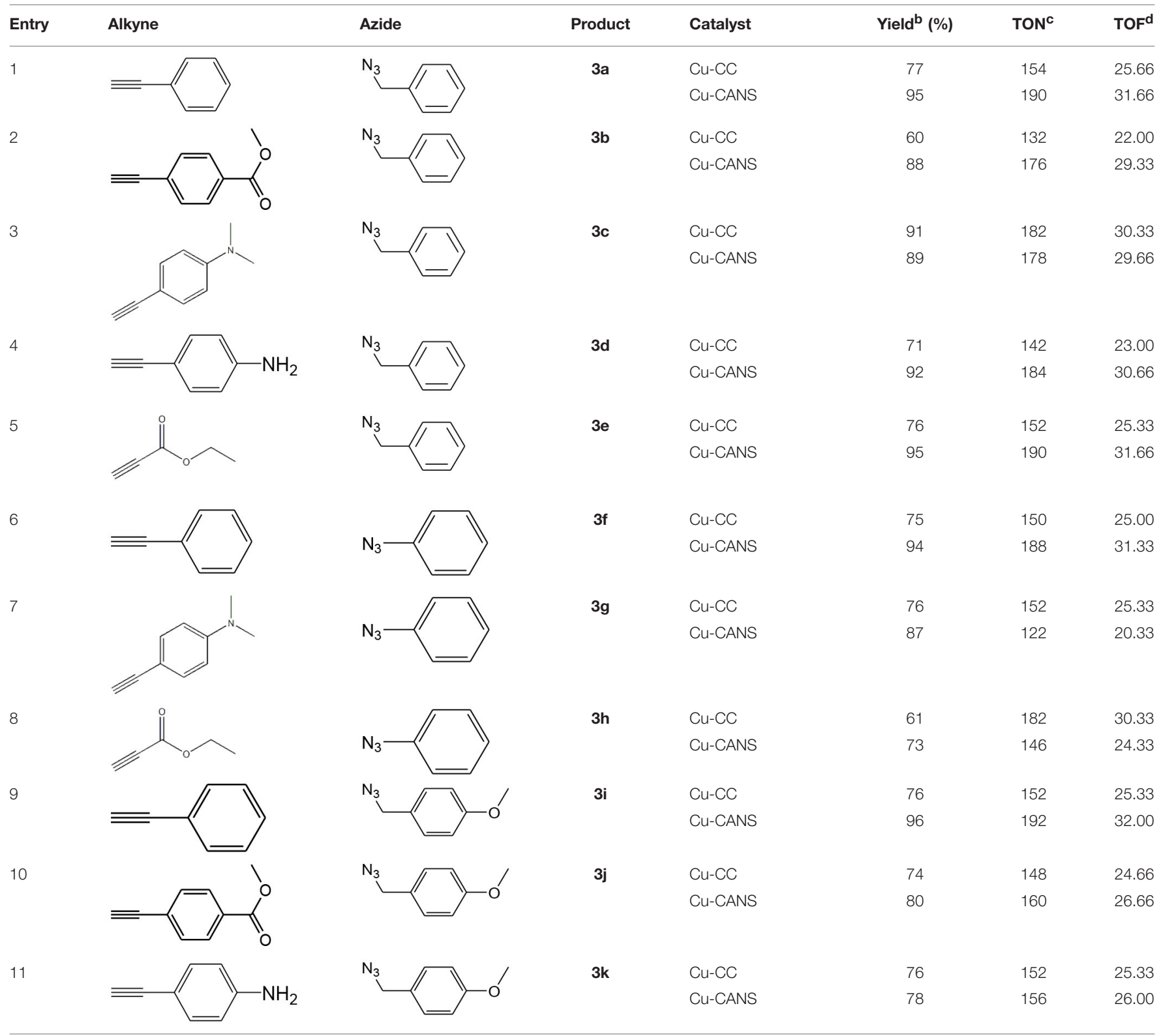

${ }^{a}$ Reaction conditions: azide ( $\left.0.75 \mathrm{mmol}\right)$; alkyne $(0.62 \mathrm{mmol})$; water $(5 \mathrm{~mL})$; catalyst (0.005 equivalent) mixed at room temperature.

${ }^{b}$ /solated yields.

${ }^{c}$ TON, Turnovers number (moles substrate/moles of catalyst).

${ }^{d}$ TOF, Turnover frequency (TON/time of reaction).

and short reaction times. Furthermore, the catalytic reaction was performed in different organic solvents, as shown in Table 3. We found that the catalytic conversion yields in water and acetonitrile were higher than those in other organic solvents, such as ethanol, methanol, toluene and hexane (entries 8-12, Table 3). As a matter of consequence, water known as a benign and inexpensive solvent, was then used as the solvent of choice.

After optimizing the reaction conditions, in order to explore the scope and generality of this protocol, several alkynes such as para-substituted aryl alkyne derivatives and activated alkyne with azides such as para-substituted benzyl and phenyl azides were used as substrates for the synthesis of 1,4-disubstituted1,2,3-triazoles. The results are given in Table 4. They show that the reactions are equally facile with both electron-donating and electron-withdrawing substituents present on the aryl alkynes and benzyl azides, as most of the reactions were completed within $6 \mathrm{~h}$, resulting one regioisomer in good to high yields of the corresponding 1,4-disubstituted-1,2,3-triazole (see Table 4). These 1,2,3-triazoles were obtained in good turnover numbers ranging from 122 to 196 . All the synthesized triazole 


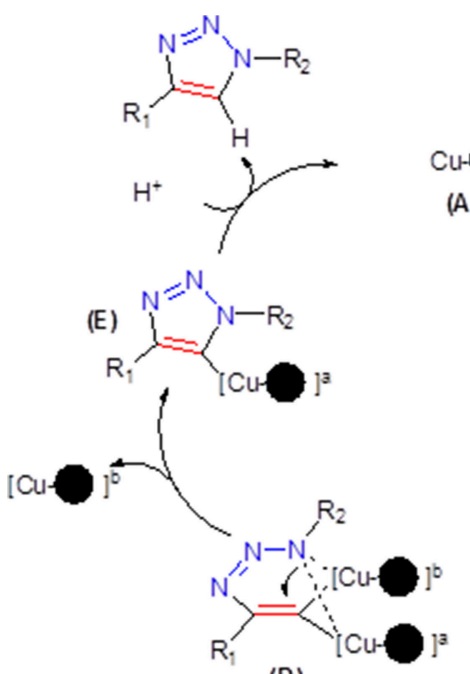

(D)
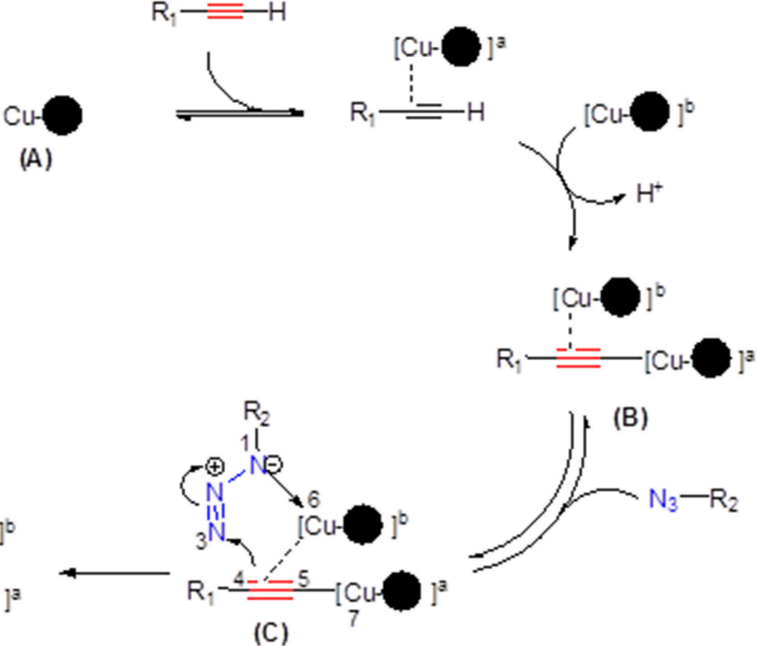

(C)

Carbon

FIGURE 6 | The proposed mechanism for the formation of 1-4-disubstituted-1,2,3-triazoles catalyzed by Cu/carbon.

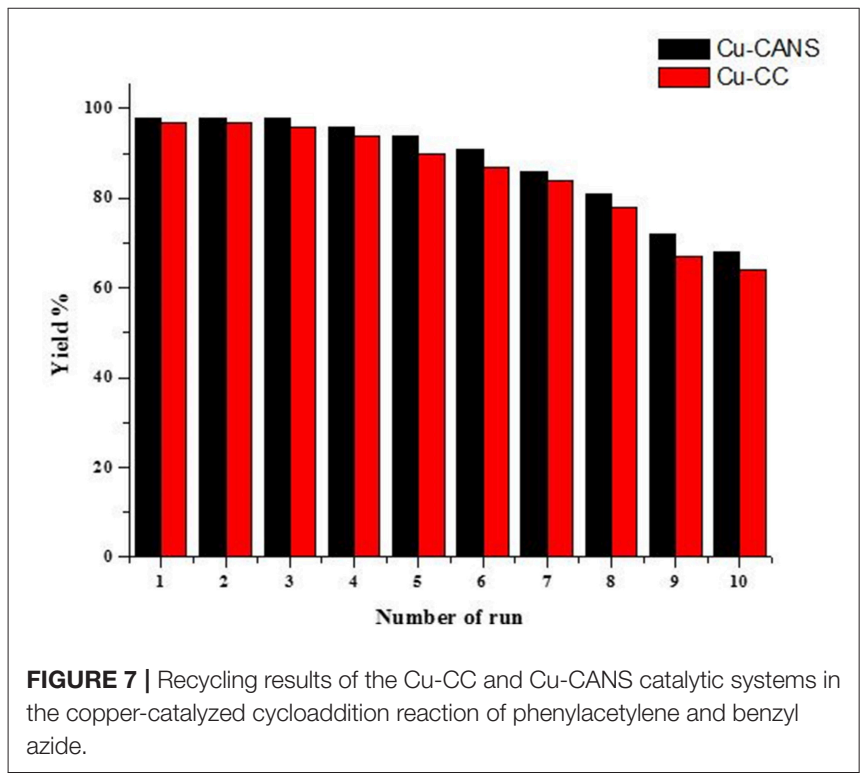

derivatives were characterized by NMR spectroscopy and HRMS analysis (see Supplementary Material).

On the basis of our previous reports (Ben El Ayouchia et al., 2018), a stepwise mechanism of CuAAC is outlined in Figure 6. The electron density of the alkyne in the proposed mechanism is reduced by the copper(I) ion stabilized in pore of the carbon support (A) forming the dinuclear copper-acetylide (B), enabling a facile nucleophilic attack by the organoazide, and then resulting in the corresponding complex $(\mathbf{C})$. The next step
TABLE 5 | Loadings of copper in each $100 \mathrm{mg}$ of copper-carbon catalyst.

\begin{tabular}{lccc}
\hline Catalyst & \multicolumn{3}{c}{ Copper loading (wt\%) } \\
\cline { 2 - 4 } & $\begin{array}{c}\text { Before } \\
\text { reaction }\end{array}$ & $\begin{array}{c}\text { After the first } \\
\text { cycle }\end{array}$ & $\begin{array}{c}\text { After the eighth } \\
\text { cycle }\end{array}$ \\
\hline Cu-CC & 3.82 & 3.65 & 0.82 \\
Cu-CANS & 1.38 & 1.09 & 0.58
\end{tabular}

consists of a nucleophilic attack at N3 of the organoazide by the acetylide carbon $\mathrm{C} 4$ forming the first covalent $\mathrm{C}-\mathrm{N}$ bond and then producing the intermediate $(\mathbf{D})$. The ring contraction of D leads to the formation of the triazolyl-copper (E). The last step corresponds to a fast protonation of the copper triazolide, releasing the final 1,2,3-triazole product as 1,4-regioisomer.

\section{Stability and Recycling of the Copper-Carbon Catalyst}

Generally copper(I) catalysts are unstable and can readily oxidize to copper(II). However, it is legitimate to question also the stability of $\mathrm{Cu}$-carbon entities as reagents in organic reactions. The conservation of the copper(I)-carbon catalysts under noninert conditions (under air), did not cause any change in their external aspects and it has been observed, unexpectedly, that after 1 year nothing has been lost of their activities. This heterogeneous catalytic system offers easy manipulation and separation by simple filtration, thus facilitating the recycling of the coppercarbon catalysts. The recyclability of copper-carbon was tested in the model cycloaddition reaction of phenylacetylene and benzyl azide (Figure 7). After completion of the cycloaddition reaction 
TABLE 6 | Comparison of the catalytic activity of cooper-carbon catalysts with others heterogeneous copper-based catalytic systems.<smiles>C#Cc1ccccc1</smiles><smiles>[N]Cc1ccccc1</smiles>

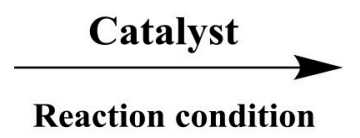

Reaction condition

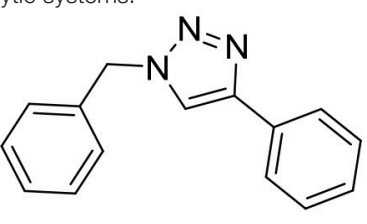

1,4-isomer

\begin{tabular}{|c|c|c|c|c|c|c|c|}
\hline Entry & Catalyst & Time (h) & $\begin{array}{l}\text { Cat. loading } \\
\text { (mol \%) }\end{array}$ & Temperature/solvent & $\begin{array}{c}\text { Number of } \\
\text { cycle }\end{array}$ & Yield (\%) & Reference \\
\hline 1 & $\mathrm{Cu}-\mathrm{CC}$ & 6 & 1 & r.t./water & 10 & 98 & This work \\
\hline 2 & Cu-CANS & 6 & 1 & r.t./water & 10 & 98 & \\
\hline 3 & $\mathrm{Cu} / \mathrm{C}$ & 48 & 10 & $23^{\circ} \mathrm{C} /$ dioxane & - & 65 & Lipshutz and Taft, 2006 \\
\hline 4 & $\mathrm{Cu}_{2} \mathrm{O} / \mathrm{C}$ & 2 & 5 & r.t./i-PrOH: $\mathrm{H}_{2} \mathrm{O}$ & 3 & 82 & López-Ruiz et al., 2012 \\
\hline 5 & $\mathrm{TRGO} / \mathrm{Cu}$ & 48 & 2 & $40^{\circ} \mathrm{C} / \mathrm{THF}$ & 4 & 99 & Shaygan Nia et al., 2014 \\
\hline 6 & Cu-Alginate & 18 & 21 & r.t./water & 3 & 98 & Rajender Reddy et al., 2007 \\
\hline 7 & Cu-Chitosan & 6 & 10 & r.t./water & 5 & 90 & Anil Kumar et al., 2015 \\
\hline 8 & $\begin{array}{l}\text { Cu- } \\
\text { Hydroxyappatite }\end{array}$ & 16 & 5 & $50^{\circ} \mathrm{C} /$ water & 8 & 95 & Masuyama et al., 2011 \\
\hline 9 & Cu-zeolite & 15 & 10 & r.t./toluene & 5 & 83 & Chassaing et al., 2007 \\
\hline
\end{tabular}

in water at room temperature, the catalyst was recovered by simple filtration and reused after washing with diethyl ether and drying in the air. The catalyst was reused directly for the next run under the same conditions. This process was performed until 10 times without any significant loss of efficiency and selectivity of the $\mathrm{Cu}$-carbon as shown by the catalytic histogram in Figure 7.

The percentages of the copper contents of the fresh and recycled $\mathrm{Cu}-\mathrm{CC}$ and $\mathrm{Cu}-\mathrm{CANS}$ after the first and the eight consecutive trial were determined by AAS analysis. The AAS results shown in Table 5 indicate that the weight percentage of the copper contents of the recycled catalysts after the first cycles is around $3.65 \mathrm{wt} \%$ for $\mathrm{Cu}-\mathrm{CC}$ and $1.01 \mathrm{wt} \%$ for $\mathrm{Cu}$-CANS, values which are lower than those of the fresh catalyst, 3.82 and $1.38 \mathrm{wt} \%$, respectively. After the eight catalytic trial, the percentages of the copper content were found to be around $0.82 \mathrm{wt} \%$ for $\mathrm{Cu}-\mathrm{CC}$ and $0.58 \mathrm{wt} \%$ for $\mathrm{Cu}-\mathrm{CANS}$. The decrease of the 1,2,3-triazole products yields is caused by the loss of the catalytically active species copper(I) during the work-up processes.

\section{Comparison of the Copper-Carbon Catalysts With Other Heterogeneous Catalysts Containing Copper Particles}

In principle, any efficient prepared catalytic system is expected be superior to the commercially available catalysts used for the same purpose. Taking into account this principle, it seemed important to compare also the catalytic activity of the copper(I)-carbon with that of other copper-containing heterogeneous catalysts among the most active known for this type of cycloaddition reaction such as $\mathrm{Cu}$-charcoal, $\mathrm{Cu}_{2} \mathrm{O}-\mathrm{C}$, copper-graphene, copperalginate, copper-chitine, copper-hydroxyappatite, copper-argile, and copper-resin (Table 6). The series of catalysts used proved to be effective since the product was obtained with excellent yields in all the cases. However, the total conversion of the substrates was possible only after $15-18 \mathrm{~h}$ of reaction, proving the lower reactivity of these catalysts in this type of reaction. Remarkably, the $\mathrm{Cu}-\mathrm{CANS}$ and $\mathrm{Cu}-\mathrm{CC}$ catalysts have a higher activity, allowing the formation of the desired product using only $0.5 \mathrm{~mol} \%$ during $6 \mathrm{~h}$ of reaction. In addition, this catalytic system has many advantages: it is robust, inexpensive, readily available, non-toxic, and has no sensitivity to humidity or air.

\section{CONCLUSIONS}

In summary, we have successfully developed a highly efficient and recyclable inexpensive copper on carbon heterogeneous catalyst for the regioselective construction of 1,4-disubstituted 1,2,3-triazoles by the [3+2] cycloaddition reactions of azides with alkynes under very strict click chemistry conditions with a sustainable fashion. The copper on carbon Cu-CANS was readily prepared by the copper(I) impregnation of carbon material that has been made from a naturally raw vegetable biomass. A wide range of azides and alkynes can be combined to form the important biologically active 1,2,3-triazoles by using $\mathrm{Cu}$ CANS in water as solvent at room temperature. Cu-CANS was recycled and reused for several catalytic trials without noticeable loss of its catalytic activity. The use of benign water as solvent at room temperature makes the entire catalytic protocol an environmental friendly one. 


\section{AUTHOR CONTRIBUTIONS}

S-ES and $\mathrm{HB}$ designed the project and supervised the synthetic and characterization chemistry works. NA and LB undertook all the synthetic experimental works and characterizations of the copper on carbon material catalysts. EE and SR carried out the synthesis of the natural carbon material originated from vegetable biomass using Argan nut shells (CANS). SR performed the characterization of the carbon precursor material CANS. S-ES, HB, MJ, SR, and HA contributed to the writing of the manuscript and interpretation of the results. MJ supplied the funding for the work.

\section{REFERENCES}

Anil Kumar, B. S. P., Harsha Vardhan Reddy, K., Karnakar, K., Satish, G. and Nageswar, Y. V. D. (2015). Copper on chitosan: an efficient and easily recoverable heterogeneous catalyst for one pot synthesis of 1,2,3-triazoles from aryl boronic acids in water at room temperature. Tetrahedron Lett. 56, 1968-1972. doi: 10.1016/j.tetlet.2015.02.107

Bahrami, K., and Arabi, M. S. (2016). Copper immobilized ferromagnetic nanoparticle triazine dendrimer (FMNP@TD-Cu(II))-catalyzed regioselective synthesis of 1,4-disubstituted 1,2,3-triazoles. New J. Chem. 40, 3447-3455. doi: 10.1039/C5NJ03219A

Bahsis, L., El Ayouchia, H. B., Anane, H., Benhamou, K., Kaddami, H., Julve, M., et al. (2018). Cellulose copper as bio-supported recyclable catalyst for the clickable azide-alkyne $[3+2]$ cycloaddition reaction in water. Inter. J. Biol. Macromol. 119, 849-856. doi: 10.1016/j.ijbiomac.2018.07.200

Ben El Ayouchia, H., Bahsis, L., Anane, H., Domingo, L. R., and Stiriba, S.-E. (2018). Understanding the mechanism and regioselectivity of the copper(I) catalyzed [2+3] cycloaddition reaction between azide and alkyne: a systematic DFT stud. RSC Adv. 8, 7670-7678. doi: 10.1039/C7RA10653J

Bock, V. D., Hiemstra, H., and van Maarseveen, J. H. (2006). CuI-catalyzed alkyneazide "click" cycloadditions from a mechanistic and synthetic perspective. Eur. J. Org. Chem. 1, 51-68. doi: 10.1002/ejoc.200500483

Bonami, L., Van Camp, W., Van Rijckegem, D., and Du Prez, F. E. (2009). Facile access to an efficient solid-supported click catalyst system based on poly(ethyleneimine). Macromol. Rapid Commun. 30, 34-38. doi: 10.1002/marc. 200800607

Buckley, B. R., Butterworth, R., Dann, S. E., Heaney, H., and Stubbs, E. C. (2015). "Copper-in-charcoal" revisited: delineating the nature of the copper species and its role in catalysis. ACS Catal. 5, 793-796. doi: 10.1021/ cs501826s

Chassaing, S., Kumarraja, M., Sani Souna Sido, A., Pale, P., and Sommer, J. (2007). Click chemistry in CuI-zeolites: the huisgen [3+2]-cycloaddition. Org. Lett. 9, 883-886. doi: 10.1021/ol0631152

Chassaing, S., Sani Souna Sido, A., Alix, A., Kumarraja, M., Pale, P., and Sommer, J. (2008). "Click chemistry" in zeolites: copper(I) zeolites as new heterogeneous and ligand-free catalysts for the huisgen [3+2] cycloaddition. Chem. Eur. J. 14, 6713-6721. doi: 10.1002/chem.200800479

Chung, C. M., Lee, S. J., Kim, J. G., and Jang, D. O. (2002). Organic-inorganic polymer hybrids based on unsaturated polyester. J. Non-Cryst. Solids 311, 195-198. doi: 10.1016/S0022-3093(02)01800-8

Coelho, A., Diz, P., Caamano, O., and Sotelo, E. (2010). Polymer-supported 1,5,7triazabicyclo[4.4.0] dec-5-ene as polyvalent ligands in the copper-catalyzed huisgen 1,3-dipolar cycloaddition. Adv. Synth. Catal. 352, 1179-1192. doi: 10.1002/adsc.200900680

Díez-Gonzalez, S., Escudero-Adan, E. C., Benet-Buchholtz, J., Stevens, E. D., Slawin, A. M. Z., and Nolan, S. P. (2010). [(NHC)CuX] complexes: synthesis, characterization and catalytic activities in reduction reactions and Click Chemistry. On the advantage of using well-defined catalytic systems. Dalton Trans. 39, 7595-7606. doi: 10.1039/c0dt00218f

\section{FUNDING}

Université Cadi Ayyad and the Spanish Ministerio de Ciencia e Innovación (MCINN) (Projects CTQ2016-75068P and Unidad de Excelencia María de Maeztu MDM2015-0538) and the Generalitat Valenciana (Project PROMETEOII/2014/070) are gratefully acknowledged for financial support.

\section{SUPPLEMENTARY MATERIAL}

The Supplementary Material for this article can be found online at: https://www.frontiersin.org/articles/10.3389/fchem. 2019.00081/full\#supplementary-material

Diz, P., Pernas, P., El Maatougui, A., Tubio, C. R., Azuaje, J., Sotelo, E., et al. (2015) Sol-gel entrapped $\mathrm{Cu}$ in a silica matrix: an efficient heterogeneous nanocatalyst for Huisgen and Ullmann intramolecular coupling reactions. Appl. Catal. A Gen. 502, 86-95. doi: 10.1016/j.apcata.2015.05.025

Freundlich, H. M. F. (1906). Over the adsorption in solution. J. Phys. Chem. 57, 385-470.

Gaetke, L. M., and Chow, C. K. (2003). Copper toxicity, oxidative stress, and antioxidant nutrients. Toxicology 189, 147-163. doi: 10.1016/S0300-483X(03)00159-8

Ghouma, I., Jeguirim, M., Sager, U., Limousy, L., Bennici, S., Däuber, E., et al. (2017). The potential of activated carbon made of agro-industrial residues in NOx immissions abatement. Energies 10:1508. doi: 10.3390/en10101508

Hong, V., Steinmetz, N. F., Manchester, M., and Finn, M. G. (2010). Labeling live cells by copper-catalyzed alkyne-azide click chemistry. Bioconjug. Chem. 10, 1912-1916. doi: $10.1021 / \mathrm{bc} 100272 \mathrm{z}$

Huisgen, R. (1963). Kinetics and mechanism of 1,3-dipolar cycloadditions. Angew. Chem. 2, 633-645. doi: 10.1002/anie.196306331

Ikotun, O. F., Marino, N., Kruger, P. E., Julve, M., and Doyle, R. P. (2010). Coordination complexes incorporating pyrophosphate: structural overview and exploration of their diverse magnetic, catalytic and biological properties. Coord. Chem. Rev. 254, 890-915. doi: 10.1016/j.ccr.2009.12.015

Jahanshahi, R., and Akhlaghinia, B. (2016). CuII immobilized on guanidinated epibromohydrin functionalized $\gamma-\mathrm{Fe}_{2} \mathrm{O}_{3} @ \mathrm{TiO}_{2}\left(\gamma-\mathrm{Fe}_{2} \mathrm{O}_{3} @ \mathrm{TiO}_{2}-\mathrm{EG}-\mathrm{CuII}\right)$ : a novel magnetically recyclable heterogeneous nanocatalyst for the green one-pot synthesis of 1,4-disubstituted 1,2,3-triazoles through alkyne-azide cycloaddition in water. RSC Adv. 6, 29210-29219. doi: 10.1039/C6RA0 $5468 \mathrm{D}$

Jumde, R. P., Evangelisti, C., Mandoli, A., Scotti, N., and Psaro, R. (2015). Aminopropyl-silica-supported $\mathrm{Cu}$ nanoparticles: an efficient catalyst for continuous-flow Huisgen azide-alkyne cycloaddition (CuAAC). J. Catal. 324, 25-31. doi: 10.1016/j.jcat.2015.01.014

Kolb, H. C., and Sharpless, K. B. (2003). The growing impact of click chemistry on drug discovery. Drug Discovery Today 8, 1128-1137. doi: 10.1016/S1359-6446(03)02933-7

Langmuir, I. (1916). The constitution and fundamental properties of solids and liquids Part I solids. J. Am. Chem. Soc. 38, 2221-2229.

Lipshutz, B. H., and Taft, B. R. (2006). Heterogeneous copper-in-charcoalcatalyzedclick chemistry. Angew. Chem. Int. Ed. 45, 8235-8238. doi: 10.1002/anie.200603726

López-Ruiz, H., de la Cerda-Pedro, E. J., Rojas-Lima, S., Pérez-Pérez, I., RodríguezSánchez, B. V., and Santillan, R. (2012). Cuprous oxide on charcoal-catalyzed ligand-free synthesis of 1,4-disubstituted 1,2,3-triazoles via click chemistry. Arkivoc 2013, 139-164. doi: 10.3998/ark.5550190.0014.312

Mark, J. E. (2006). Some novel polymeric nanocomposites. Acc. Chem. Res. 39, 881-888. doi: 10.1021/ar040062k

Masuyama, Y., Yoshikawa, K., Suzuki, N., Hara, K., and Fukuoka, A. (2011). Hydroxyapatite-supported copper(II)-catalyzed azide-alkyne [3+2] cycloaddition with neither reducing agents nor bases in water. Tetrahedron Lett. 52, 6916-6918. doi: 10.1016/j.tetlet.2011.10.060 
Miao, T., and Wang, L. (2008). Regioselective synthesis of 1,2,3-triazoles by use of a silica-supported copper(I) catalyst. Synthesis 3, 363-368. doi: $10.1055 / \mathrm{s}-2008-1032037$

Moghaddam, F. M., and Ayati, S. E. (2015). Copper immobilized onto a triazole functionalized magnetic nanoparticle: a robust magnetically recoverable catalyst for "click" reactions. RSC Adv. 5, 3894-3902. doi: 10.1039/C4RA13330G

Ostapenko, N., Dovbeshko, G., Kozlova, N., Suto, S., and Watanabe, A. (2008). Conformation change of nanosized silicon-organic polymer oriented into ordered nanoporous silicas. Thin Solid Films 516, 8944-8948. doi: 10.1016/j.tsf.2007.11.069

Pachón, L. D., van Maarseveen, J. H., and Rothenberg, G. (2005). Click chemistry: copper clusters catalyse the cycloaddition of azides with terminal alkynes. $A d v$. Synth. Catal. 347, 811-815. doi: 10.1002/adsc.200404383

Pourjavadi, A., Motamedi, A., Hosseini, S. H., and Nazari, M. (2016). Magnetic starch nanocomposite as a green heterogeneous support for immobilization of large amounts of copper ions: heterogeneous catalyst for click synthesis of 1,2,3-triazoles. RSC Adv. 6, 19128-19135. doi: 10.1039/C5RA25519H

Pourjavadi, A., Tajbakhsh, M., Farhang, M., and Hosseini, S. H. (2015). Copper-loaded polymeric magnetic nanocatalysts as retrievable and robust heterogeneous catalysts for click reactions. New J. Chem. 39, 4591-4600. doi: 10.1039/C4NJ02134G

Rajender Reddy, K., Rajgopal, K., and Lakshmi Kantam, M. (2007). Copperalginates: a biopolymer supported $\mathrm{Cu}$ (II) catalyst for 1,3-dipolar cycloaddition of alkynes with azides and oxidative coupling of 2-naphthols and phenols in water. Catal. Lett. 114, 36-40. doi: 10.1007/s10562-007-9032-x

Run, M., Wu, S. Z., Zhang, D. Y., and Wu, G. (2007). A polymer/mesoporous molecular sieve composite: preparation, structure and properties. Mater. Chem. Phys. 105, 341-347. doi: 10.1016/j.matchemphys.2007.04.070

Samim, M., Kaushik, N. K., and Maitra, A. (2007). Effect of size of copper nanoparticles on its catalytic behaviour in Ullman reaction. Bull. Mater. Sci. 30, 535-540. doi: 10.1007/s12034-007-0083-9

Shaygan Nia, A., Rana, S., Döhler, D., Noirfalise, X., Belfiore, A., and Binder, W. H. (2014). Click chemistry promoted by graphene supported copper nanomaterials. Chem. Commun. 50, 15374-15377. doi: 10.1039/C4CC07774A

Tajbakhsh, M., Farhang, M., Baghbanian, S. M., Hosseinzadeh, R., and Tajbakhs, M. (2015). Nano magnetite supported metal ions as robust, efficient and recyclable catalysts for green synthesis of propargylamines and 1,4-disubstituted 1,2,3triazoles in water. New J. Chem. 39, 1827-1839. doi: 10.1039/C4NJ01866D

Tornøe, C. W., Christensen, C., and Meldal, M. (2002). Peptidotriazoles on solid phase: [1,2,3]-triazoles by regiospecific copper(I)-catalyzed 1,3-dipolar cycloadditions of terminal alkynes to azides. J. Org. Chem. 67, 3057-3064. doi: $10.1021 /$ jo011148j
Wallyn, S., Lammens, M., O’Reilly, R. K., and Du Prez, F. (2011). Highly active, thermo-responsive polymeric catalytic system for reuse in aqueous and organic CuAAC reactions. J. Polym. Sci. Part A Polym. Chem. 49, 2878-2885. doi: $10.1002 /$ pola. 24723

Xiong, X., and Cai, L. (2013). Application of magnetic nanoparticle-supported $\mathrm{CuBr}$ : a highly efficient and reusable catalyst for the one-pot and scale-up synthesis of 1,2,3-triazoles under microwave-assisted conditions. Catal. Sci. Technol. 3, 1301-1307. doi: 10.1039/C3CY20680GS

Xiong, X., Jiang, Y., Xiao, S., Shi, L., and Song, S. (2016). Highly efficient synthesis of 1,2,3-triazoles catalyzed by Schiff base functionalized chitosanCuBr catalyst. Chem. J. Chin. Univ. 37, 1863-1869. doi: 10.7503/cjcu201 60252

Yadav, J. S., Subba Reddy, B. V., Madhusudhan Reddy, G., and Narasimha Chary, D. (2007). Three component, regioselective, one-pot synthesis of $\beta$ hydroxytriazoles from epoxides via 'click reactions'. Tetrahedron Lett. 48 8773-8776. doi: 10.1016/j.tetlet.2007.09.160

Yan, Z.-Y., Zhao, Y.-B., Fan, M.-J., Liu, W.-M., and Liang, Y.-M. (2005). General synthesis of (1-substituted-1H-1,2,3-triazol-4-ylmethyl)-dialkylamines via a copper(I)-catalyzed three-component reaction in water. Tetrahedron 61, 9331-9337. doi: 10.1016/j.tet.2005.07.060

Yang, M., Jalloh, A. S., Wei, W., Zhao, J., Wu, P., and Chen, P. R. (2014). Biocompatible click chemistry enabled compartment-specific $\mathrm{pH}$ measurement inside E. coli. Nat. Commun. 5, 4981-5024. doi: 10.1038/ncomms 5981

Zhang, X., Li, H., You, L., Tang, Y., and Hsung, R. P. (2006). Copper salt-catalyzed azide-[3+2] cycloadditions of ynamides and bis-ynamides. Adv. Synth. Catal. 348, 2437-2442. doi: 10.1002/adsc.200600404

Zheng, J., Li, G., Xingfa, M., Wang, Y., Wu, G., and Cheng, Y. (2008). Polyaniline- $\mathrm{TiO}_{2}$ nano-composite-based trimethylamine QCM sensor and its thermal behavior studies. Sens. Actuators B Chem. 133, 374-380. doi: 10.1016/j.snb.2008.02.037

Conflict of Interest Statement: The authors declare that the research was conducted in the absence of any commercial or financial relationships that could be construed as a potential conflict of interest.

Copyright (C) 2019 Aflak, Ben El Ayouchia, Bahsis, El Mouchtari, Julve, Rafqah, Anane and Stiriba. This is an open-access article distributed under the terms of the Creative Commons Attribution License (CC BY). The use, distribution or reproduction in other forums is permitted, provided the original author(s) and the copyright owner(s) are credited and that the original publication in this journal is cited, in accordance with accepted academic practice. No use, distribution or reproduction is permitted which does not comply with these terms. 